
\title{
R Research Soure \\ Higher-Order Kinematics Modeling of 3-RRS Parallel Mechanism Based on CGA
}

\section{Chang Wang \\ Yanshan University \\ Erwei Li \\ Yanshan University \\ Yanzhi Zhao \\ Yanshan University \\ Hui Bian \\ Yanshan University \\ Mingchao Geng \\ Yanshan University}

Tieshi Zhao ( $\nabla$ tszhao@ysu.edu.cn )

Yanshan University https://orcid.org/0000-0003-3491-839X

\section{Original Article}

Keywords: CGA, Flag, Rigid body framing, Higher-order kinematics, 3-RRS parallel mechanism

Posted Date: November 6th, 2020

DOl: https://doi.org/10.21203/rs.3.rs-101801/v1

License: (c) (1) This work is licensed under a Creative Commons Attribution 4.0 International License.

Read Full License 


\section{Title page}

\section{Higher-order kinematics modeling of 3-RRS parallel mechanism based on CGA}

Chang Wang, born in 1988, is currently a PhD candidate at Parallel Robot and Mechatronic System Laboratory, Yanshan University, China. He received his master degree from Yanshan University, China, in 2014. His research interests include theory and application of parallel manipulators, and ship motion compensation technology.

E-mail: wangchangys@ @stumail.ysu.edu.cn

Tie-Shi Zhao, born in 1963, is currently a professor and a PhD candidate supervisor at Parallel Robot and Mechatronic System Laboratory, Yanshan University, China. He received his PhD degree from Yanshan Universtiy, China, in 2000. His research interests include theory and application of parallel manipulators, wearable rehabilitation robot and ship motion compensation technology.

E-mail: tszhao@ysu.edu.cn

Er-Wei Li, born in 1987, is currently a lecturer at Parallel Robot and Mechatronic System Laboratory, Yanshan University, China. He received his $\mathrm{PhD}$ degree from Yanshan Universtiy, China, in 2018. His research interests include theory and application of parallel manipulators, and ship motion compensation technology.

E-mail: lierwei@ysu.edu.cn

Yan-Zhi Zhao, born in 1981, is currently a professor and a PhD candidate supervisor at Parallel Robot and Mechatronic System Laboratory, Yanshan University, China. He received his $\mathrm{PhD}$ degree from Yanshan Universtiy, China, in 2009. His research interests include theory and application of parallel manipulators, and rescuing robot.

E-mail: yzzhao@ysu.edu.cn

Hui Bian, born in 1982, is currently an associate professor at Parallel Robot and Mechatronic System Laboratory, Yanshan University, China. He received his PhD degree from Yanshan Universtiy, China, in 2011. His research interests include theory and application of parallel manipulators, and wearable rehabilitation robot.

E-mail: ysubh@ysu.edu.cn

Ming-Chao Geng, born in 1984, is currently a lecturer at School of Mechanical Engineering, Hebei University of Architecture, China. He received his $\mathrm{PhD}$ degree from Yanshan Universtiy, China, in 2015. His research interests include parallel robots and multi-bodies system dynamics.

E-mail: gmingchao@163.com

\section{Corresponding author: Tie-Shi Zhao E-mail: tszhao@ysu.edu.cn}




\title{
Higher-order kinematics modeling of 3-RRS parallel mechanism
}

\author{
Chang Wang ${ }^{1,2} \cdot$ Tie-Shi Zhao ${ }^{1,2} \cdot$ Er-Wei Li ${ }^{1,2} \cdot$ Yan-Zhi Zhao ${ }^{1,2} \cdot$ Hui Bian ${ }^{1,2} \cdot$ Ming-Chao Geng $^{3}$
}

Received June xx, 201x; revised February xx, 201x; accepted March xx, 201x

(c) Chinese Mechanical Engineering Society and Springer-Verlag Berlin Heidelberg 2017

\begin{abstract}
Higher-order kinematics of mechanisms has been applied in servo motor control, human-robot interaction and machinery life design fields, etc. The representations of acceleration and jerk by screws have been fully developed by researchers with the methods of the differential of the matrix representation of $S E(3)$ group. Clifford algebra, which is tighter and with higher computational efficiency than the matrix method, is another representation of the motions of rigid bodies. It has been used in position kinematics, grub task motion planning, and robot vision for its convenience of geometric representations and calculations. As far as we know, the work of higher-order kinematics of mechanisms based on Clifford algebra is rare. First, after recalling the based theory of motion representation in conformal geometric algebra (CGA), the mathematical relationships between flag and motor are built. Second, a method for the higher-order kinematics modeling of serial chain mechanisms is proposed. Finally, the higher-order kinematics of the 3-RRS parallel mechanism is built to prove the correctness of the algorithm. This work further enriches the application of CGA for the higher-order kinematics modeling of parallel mechanisms. Keywords: CGA • Flag • Rigid body framing - Higher-order kinematics $\bullet$-RRS parallel mechanism
\end{abstract}

\section{Introduction}

Pose (position and orientation), velocity, and acceleration

Tie-Shi Zhao

tszhao@ysu.edu.cn

1 Parallel Robot and Mechatronic System Laboratory, Yanshan University, Qinhuangdao 066004, China

2 Key Laboratory of Advanced Forging \& Stamping Technology and Science of Ministry of National Education, Yanshan University, Qinhuangdao 066004, China

3 School of Mechanical Engineering, Hebei University of Architecture, Zhangjiakou 075000, China calculations are the main topics of kinematics of mechanisms, and they play an essential role in motion planning, control, evaluation of robot $[1,2]$, etc. The main task of velocity analysis, which is also called the first-order kinematics, is to obtain Jacobian matrix that represents the mapping of velocities between actuators and output body. Acceleration analysis, which has relationships with the Hessian matrix, is mainly used to obtain the mapping of accelerations between actuators and output body. Second-order kinematics is the base for dynamic modeling of mechanisms. In recent years, third-order kinematics of mechanism, which is the mapping between jerks of actuators and the output, has attracted researchers. Compared with first- and second-order kinematics, jerk analysis seems less important. However, in some applications, such as servo control of motors[3], motion planning[4, 5], human-robot interaction[6], elevator control[7], and design of rollercoasters[8], third-order kinematics is also needed to be considered, for it has impacts on electron device, vibration of mechanisms, and body feelings[9]. For instance, if the jerk of a robot is large during fast motions, the acceleration changes frequently and will lead to premature wear of joints or actuators. In another case, if the jerk of a human body is large, the muscles of the body may fail to take actions to the change of the acceleration, and it could cause discomfort or even damage the human tissues. Higher-order kinematics modeling is also used to analyze the instantaneous mobility and the cusp of some mechanisms [10-14].

Screw theory, Lie group, and Lie algebra have been applied a lot in kinematics and dynamics modeling of mechanisms, especially the spatial multi-loop mechanisms. Screw theory is built in the 19th century. Plücker first used six coordinates to represent a line in $3 \mathrm{D}$ space. It was Ball 
who first systematically built screw theory, and give guidance to the application of screw theory in the field of rigid body kinematics and dynamics[15]. From then on, a lot of researchers, such as Hunt[16], Waldron[17], Suginmoto[18], Duffy[19], Phillips[20], Selig[21], Dai[22] and Huang[23], have devoted themselves to the application and development of this tool. Until now, screw theory has been used in synthesis, kinematics, dynamics, and control of all kinds of mechanisms. Screws were initially used to represent the instantaneous motion or the force with the rigid bodies. Then Mises[24] defined the cross product and pointed out the necessity to take the derivative of screws with time. Brand [25] clearly defined acceleration motor as the derivation of velocity screw. Lie group and Lie algebra were developed paralleled with Ball's work, and it was Karger who developed the relationships between screw and Lie algebra and revealed that velocity screw is the element of $s e(3)$ and wrench is the element of $s e^{*}(3)$ [26]. Based on these developments, a more general Newton-Euler equation was discovered for the modeling of rigid body dynamics [27-29] and was used for kinematics and dynamics modeling of mechanisms. Higher-order kinematics of mechanisms based on these theories have also been significantly developed $[11,30]$.

The matrix representation of $S E(3)$, which is also called transformation matrix, is usually used to represent motions of rigid bodies in three-dimensional space. Based on the differential of transformation matrix, the screw velocity represented by Lie algebra can then be obtained. However, using a $4 \times 4$ matrix to describe the six-dimension motions of a rigid body has redundancy and will generate limitations in the derivation or calculation process. Compared with the matrix-vector represent of motions proposed by Gibbs, the geometric algebra methods proposed by Clifford have some other superiorities, such as the tightness of the representation and the higher calculation efficiency. For instance, some commercial software use the quaternions to represent the rotation to avoid the orthogonality error correction caused by numerical errors. The conformal group of space in Clifford algebra has been taken attention by Angles in the 1980s[31, 32], whose recently work about the conformal spin structure can be obtained in [33, 34]. Hestenes made some promotion of Clifford algebra (or geometric algebra) in terms of physical computing[35]. The conformal geometric algebra (CGA) introduced by $\mathrm{Li}$ and Hestenes [36] have been used to solve problems in vision, kinematics, and dynamics of robots due to its convenience and efficiency when representing points, lines, circles, spheres and planes. Ten years ago, Hestenes proposed the method to solve the kinematics and dynamics of open chain kinematics and give the definition of screws and wrenches represented with Clifford algebra and CGA[37]. At the same time, Selig also proposed a method using an eight-dimensional algebra to build rigid body dynamics, and he claimed that inertias, velocities, and momenta all can be represented as the elements of that algebra and all the relationships between physical quantities could be given by the standard operations[38]. After that, attentions start to be paid by researchers in the robotic fields and amount of work has been done based on Clifford algebra[39, 40], such as the forward and inverse position kinematics[41-44], singularity analysis[45, 46], robot POC representation[47], type synthesis of parallel mechanisms[48], dynamics[49], freedom or constraint analysis[50-52], first-order kinematics and grub jobs[53]. As far as we know, the research of higher-order kinematics of parallel mechanisms based on CGA is relatively rare.

This research extends the application of CGA to mechanistic kinematics so that the positional solution and higher-order kinematics of mechanisms can be uniformly described in the same algebra system. Section 2 briefly gives a recall of Clifford algebra and CGA. Section 3 introduces the rotation, translation, and screw motion in the algebra, and introduces the framing method of rigid body based on flag. The analytic relationships between flag and motor is first revealed. Section 4 deduces the first-, second-, and third-order kinematics of serial chain, and the iteration formulas are given. Section 5 provides the modeling method of the position calculation and the higher-order kinematics of the 3-RRS parallel mechanism. Conclusions are drawn in Section 6.

We also refers to $[35,54-56]$ for the based theory and calculation of Clifford algebra, and the programs in this paper are built based on the project of Clifford algebra[57].

\section{CGA foundations}

The five-dimensional CGA, which uses three Euclidean basis vectors $. \boldsymbol{e}_{1}, \boldsymbol{e}_{2}, \boldsymbol{e}_{3}$. and two additional basis vectors $\boldsymbol{e}_{4}, \boldsymbol{e}_{5}$ with positive and negative signatures, respectively, is represented by $\mathbb{G}^{4,1}$. The operation rules of the basis vectors are listed as follows:

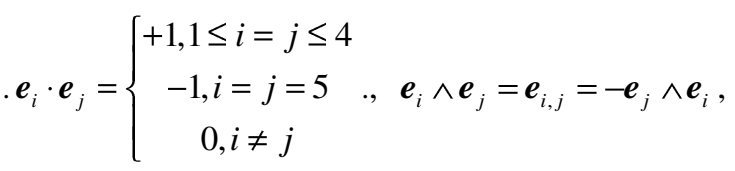

where - is inner product and $\wedge$ is outer product. Another two bases are $\boldsymbol{e}_{\infty}$ and $\boldsymbol{e}_{0}$, with definitions by 
$\boldsymbol{e}_{\infty}=\boldsymbol{e}_{4}+\boldsymbol{e}_{5}$ and $\boldsymbol{e}_{0}=\frac{1}{2} \boldsymbol{e}_{5}-\boldsymbol{e}_{4}$. There are 32 special blades of $\mathbb{G}^{4,1}$ and their linear combinations are called multi-vectors. A k-blade is denoted by $\boldsymbol{A}_{\langle k\rangle}$ that can be represented by the outer products of $k$ linear independent 1-vectors. Besides outer product and inner product, geometric product of blades is also frequently used, and it is defined as

$$
\boldsymbol{A}_{\langle k\rangle} \boldsymbol{A}_{\langle l\rangle}=\left\langle\boldsymbol{A}_{\langle k\rangle} \boldsymbol{A}_{\langle l\rangle}\right\rangle_{|k-l|}+\left\langle\boldsymbol{A}_{\langle k\rangle} \boldsymbol{A}_{\langle l\rangle}\right\rangle_{|k-l|+2}+\cdots\left\langle\boldsymbol{A}_{\langle k\rangle} \boldsymbol{A}_{\langle l\rangle}\right\rangle_{|k+l|},
$$

where.$\langle\bullet\rangle_{m}$. denotes the element of $m$ grade, $\left\langle\boldsymbol{A}_{\langle k\rangle} \boldsymbol{A}_{\langle l\rangle}\right\rangle_{|k-l|}$ is the inner product $\boldsymbol{A}_{\langle k\rangle} \cdot \boldsymbol{A}_{\langle l\rangle}$, and $\left\langle\boldsymbol{A}_{\langle k\rangle} \boldsymbol{A}_{\langle l\rangle}\right\rangle_{|k+l|}$ is the outer product $\boldsymbol{A}_{\langle k\rangle} \wedge \boldsymbol{A}_{\langle l\rangle}$. More detail can be found in Charter 3 of [55]. The geometric product of two multi-vectors can also be expressed by the combinations of the products of blades according to the distributive property of the geometric product.

Conformal geometric entities can be represented by both inner product null space (IPNS) and outer product null space(OPNS) in CGA $[36,55] \ldots \boldsymbol{P}, \boldsymbol{\Pi}, \boldsymbol{S}, \boldsymbol{C}, \boldsymbol{L}$ and $\boldsymbol{P} \boldsymbol{p}$ denote the IPNS representations of point, plane, sphere, circle, line and point pair, respectively. OPNS representation is denoted by adding the superscript *, e.g., $\boldsymbol{\Pi}^{*}$ is a plane, as shown in Table 1. Moreover, $\boldsymbol{F}, \boldsymbol{M}$, $\boldsymbol{R}, \boldsymbol{T}$ and $\boldsymbol{V}$ represent the flag, motor, rotor, translator and screw, respectively. A point (or a vector) in three-dimensional space is $\boldsymbol{p}=p_{1} \boldsymbol{e}_{1}+p_{2} \boldsymbol{e}_{2}+p_{3} \boldsymbol{e}_{3}$, and there exists a mapping from $\boldsymbol{p}$ to the point in conformal space, i.e.

$$
\boldsymbol{P}=\mathcal{P}(\boldsymbol{p})=\boldsymbol{p}+\frac{1}{2} \boldsymbol{p}^{2} \boldsymbol{e}_{\infty}+\boldsymbol{e}_{o}
$$

Its inverse mapping is $\boldsymbol{p}=\mathcal{P}^{-1}(\boldsymbol{P})$.

On the other hand, the vectors and matrices are represented by standard bold letters, i.e., $\mathbf{J}$ is Jacobian matrix and $\boldsymbol{\theta}$ is a column vector of joint angles.

Table 1 Representations of conformal geometric entities

\begin{tabular}{|c|c|c|}
\hline & the middle plane of $\boldsymbol{P}_{1}, \boldsymbol{P}_{2}$. & \\
\hline Sphere & $\begin{array}{l}\boldsymbol{S}=\boldsymbol{P}-\frac{1}{2} r^{2} \boldsymbol{e}_{\infty}, \text { where } r \text { is } \\
\text { the diameter of the sphere, } \boldsymbol{P} \\
\text { is its center. }\end{array}$ & $\begin{array}{l}\boldsymbol{S}^{*}=\boldsymbol{P}_{1} \wedge \boldsymbol{P}_{2} \wedge \boldsymbol{P}_{3} \wedge \boldsymbol{P}_{4}, \\
\text { where } \boldsymbol{P}_{1}, \boldsymbol{P}_{2}, \boldsymbol{P}_{3}, \quad \boldsymbol{P}_{4} \\
\text { are four arbitrary points on } \\
\text { the sphere. }\end{array}$ \\
\hline Circle & $\begin{array}{l}C=S_{1} \wedge S_{2} \text {, where } C \text { is the } \\
\text { intersection circle of the two } \\
\text { spheres } S_{1} \text { and } S_{2} \text {. }\end{array}$ & $\begin{array}{l}\boldsymbol{C}^{*}=\boldsymbol{P}_{1} \wedge \boldsymbol{P}_{2} \wedge \boldsymbol{P}_{3} \text {, where } \\
\boldsymbol{P}_{1}, \quad \boldsymbol{P}_{2}, \quad \boldsymbol{P}_{3} \text { are three } \\
\text { arbitrary points on the } \\
\text { circle. }\end{array}$ \\
\hline Line & $\begin{array}{l}\boldsymbol{L}=\boldsymbol{\Pi}_{1} \wedge \boldsymbol{\Pi}_{2} \text {, where } \boldsymbol{L} \text { is the } \\
\text { intersection line of the two } \\
\text { planes } \boldsymbol{\Pi}_{1} \text { and } \boldsymbol{\Pi}_{2} .\end{array}$ & $\begin{array}{l}\boldsymbol{L}^{*}=\boldsymbol{P}_{1} \wedge \boldsymbol{P}_{2} \wedge \boldsymbol{e}_{\infty}, \text { where } \\
\boldsymbol{P}_{1} \text { and } \boldsymbol{P}_{2} \text { are any two } \\
\text { points on the line; or, } \\
\boldsymbol{L}^{*}=\boldsymbol{C}^{\wedge} \boldsymbol{e}_{\infty}, \text { where } \boldsymbol{L}^{*} \\
\text { is the line passing through } \\
\text { the center and being norm } \\
\text { to the face of a circle } \boldsymbol{C} .\end{array}$ \\
\hline $\begin{array}{l}\text { Point } \\
\text { pair }\end{array}$ & $\begin{array}{l}P p=S_{1} \wedge S_{2} \wedge S_{3}=C^{\wedge} S_{3}, \\
\text { point pair determined by three } \\
\text { spheres, or determined by a } \\
\text { circle and a sphere. }\end{array}$ & $\boldsymbol{P p}^{*}=\boldsymbol{P}_{1}^{\wedge} \boldsymbol{P}_{2}$ \\
\hline
\end{tabular}

\begin{tabular}{l|l|l}
\hline Entity & IPNS representation & OPNS representation \\
\hline Point & $\boldsymbol{P}=\mathrm{P}(\boldsymbol{p})=\boldsymbol{p}+\frac{1}{2} \boldsymbol{p}^{2} \boldsymbol{e}_{\infty}+\boldsymbol{e}_{o}$ & \\
\hline Plane & $\boldsymbol{\Pi}=\boldsymbol{n}+d \boldsymbol{e}_{\infty}$, where $\boldsymbol{n}$ is the \\
& $\begin{array}{l}\text { direction and } d \text { is the } \\
\text { distance from the origin of the } \\
\text { plane; } \\
\boldsymbol{\Pi}=\boldsymbol{P}_{1}-\boldsymbol{P}_{2}, \text { where } \boldsymbol{\Pi} \text { is }\end{array}$ & $\begin{array}{l}\text { where } \boldsymbol{P}_{1} \wedge \boldsymbol{P}_{2} \wedge \boldsymbol{P}_{3} \wedge \boldsymbol{P}_{\infty}, \boldsymbol{P}_{3} \text { are } \\
\text { three arbitrary points } \\
\left(\text { except } \boldsymbol{e}_{\infty}\right) \text { on the plane. }\end{array}$ \\
\hline
\end{tabular}

\section{Transformation and differential motion of conformal geometric entities and rigid bodies}

\subsection{Transformation of geometric entities}

\subsubsection{Rotation about the axis passing through origin}

The IPNS representation of two planes that passing through the origin are $\boldsymbol{\Pi}_{1}=\overline{\boldsymbol{m}}, \quad \boldsymbol{\Pi}_{2}=\overline{\boldsymbol{n}}$, where $\overline{\boldsymbol{m}}=m_{1} \boldsymbol{e}_{1}+m_{2} \boldsymbol{e}_{2}+m_{3} \boldsymbol{e}_{3}$ and $\overline{\boldsymbol{n}}=n_{1} \boldsymbol{e}_{1}+n_{2} \boldsymbol{e}_{2}+n_{3} \boldsymbol{e}_{3} \quad$ are both unit vector, and the angle between them is $\theta / 2$, as shown in Figure 1. If $\boldsymbol{A}_{\langle k\rangle}$ reflects with $\boldsymbol{\Pi}_{2}$ and $\boldsymbol{\Pi}_{1}$ sequentially, then it rotates about the intersecting line of these two planes with the angle $\theta$. The rotation result is

$$
\boldsymbol{A}_{\langle k\rangle}^{\prime}=\boldsymbol{R} \boldsymbol{L}_{0}, \theta \quad \boldsymbol{A}_{\langle k\rangle} \tilde{\boldsymbol{R}} \boldsymbol{L}_{0}, \theta
$$

with the definitions

$$
\left\{\begin{aligned}
\boldsymbol{R} \boldsymbol{L}_{0}, \theta=\overline{\boldsymbol{m}} \overline{\boldsymbol{n}} & =\cos \left(\frac{\theta}{2}\right)-\boldsymbol{L}_{0} \sin \left(\frac{\theta}{2}\right) \\
\boldsymbol{L}_{o} & =\frac{\overline{\boldsymbol{n}} \wedge \overline{\boldsymbol{m}}}{\|\overline{\boldsymbol{n}} \wedge \overline{\boldsymbol{m}}\|}
\end{aligned}\right.
$$

where $\boldsymbol{R} \boldsymbol{L}_{0}, \theta$ is a rotor, $\tilde{\boldsymbol{R}} \boldsymbol{L}_{0}, \theta$ is the reverse of $\boldsymbol{R} \boldsymbol{L}_{0}, \theta, \boldsymbol{L}_{0}$ is the intersecting line of these two planes. $\boldsymbol{R} \boldsymbol{L}_{0}, \theta$ is usually abbreviated as $\boldsymbol{R}_{0}$. The unit vector of the direction of $\boldsymbol{L}_{0}$ is $\overline{\boldsymbol{\omega}}$ which can be obtained by

$$
\overline{\boldsymbol{\omega}}=-\boldsymbol{L}_{0} \boldsymbol{e}_{1,2,3},
$$


where $\overline{\boldsymbol{\omega}}=\omega_{1} \boldsymbol{e}_{1}+\omega_{2} \boldsymbol{e}_{2}+\omega_{3} \boldsymbol{e}_{3}$. On the other hand, $\boldsymbol{L}_{0}$ can be represented by $\overline{\boldsymbol{\omega}}$ as follows:

$$
\boldsymbol{L}_{0}=\overline{\boldsymbol{\omega}} \boldsymbol{e}_{1,2,3}=\omega_{3} \boldsymbol{e}_{1,2}-\omega_{2} \boldsymbol{e}_{1,3}+\omega_{1} \boldsymbol{e}_{2,3}
$$

Three special rotors are listed as follows:

1) The rotor about $\boldsymbol{e}_{3}$ is $\boldsymbol{R}_{3} \alpha=\cos \left(\frac{\alpha}{2}\right)-\boldsymbol{e}_{1,2} \sin \left(\frac{\alpha}{2}\right)$, where $\alpha$ is the rotation angle.

2) The rotor about $\boldsymbol{e}_{2}$ is $\boldsymbol{R}_{2} \beta=\cos \left(\frac{\beta}{2}\right)+\boldsymbol{e}_{1,3} \sin \left(\frac{\beta}{2}\right)$, where $\beta$ is the rotation angle.

3) The rotor about $\boldsymbol{e}_{1}$ is $\boldsymbol{R}_{1} \gamma=\cos \left(\frac{\gamma}{2}\right)-\boldsymbol{e}_{2,3} \sin \left(\frac{\gamma}{2}\right)$, where $\gamma$ is the rotation angle.

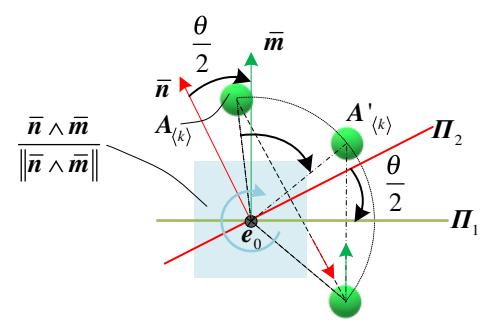

Figure 1 Rotation

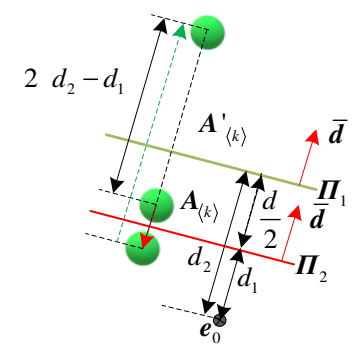

Figure 2 Translation

\subsubsection{Translation}

The IPNS representation of two parallel planes are $\boldsymbol{\Pi}_{1}=\overline{\boldsymbol{d}}+d_{1} \boldsymbol{e}_{\infty}$ and $\boldsymbol{\Pi}_{2}=\overline{\boldsymbol{d}}+d_{2} \boldsymbol{e}_{\infty}$, where $\overline{\boldsymbol{d}}$ is the normal unit vector of them. If $\boldsymbol{A}_{\langle k\rangle}$ reflect with $\boldsymbol{\Pi}_{2}$ and $\boldsymbol{\Pi}_{1}$ sequentially, then it translates along $\overline{\boldsymbol{d}}$ with the length $d=2\left(d_{2}-d_{1}\right)$, the translator of this motion is

$$
\boldsymbol{T} \overline{\boldsymbol{d}}, d=\boldsymbol{\Pi}_{2} \boldsymbol{\Pi}_{1}=1-\left(d_{2}-d_{1}\right) \overline{\boldsymbol{d}} \boldsymbol{e}_{\infty}=1-\frac{d}{2} \overline{\boldsymbol{d}} \boldsymbol{e}_{\infty} .
$$

The translation result of $\boldsymbol{A}_{\langle k\rangle}$ is

$$
\boldsymbol{A}_{\langle k\rangle}^{\prime}=\boldsymbol{T} \overline{\boldsymbol{d}}, d \quad \boldsymbol{A}_{\langle k\rangle} \tilde{\boldsymbol{T}} \overline{\boldsymbol{d}}, d .
$$

\subsubsection{Screw motion}

Screw can usually represents the motions of joints of spatial mechanisms, and it can make geometric entities rotate about an axis with an angle $\theta$ and translate along the same axis with a distance $h \theta$, where $h$ is pitch.

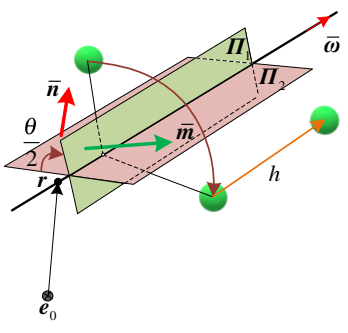

Figure 3 Screw motion

As shown in Figure 3, the intersecting line of two planes $\boldsymbol{\Pi}_{1}=\overline{\boldsymbol{m}}+d_{1} \boldsymbol{e}_{\infty} \quad$ and $\quad \boldsymbol{\Pi}_{2}=\overline{\boldsymbol{n}}+d_{2} \boldsymbol{e}_{\infty} \quad$ is $\boldsymbol{L}=\boldsymbol{\Pi}_{2} \wedge \boldsymbol{\Pi}_{1}$. The angle of these two planes is $\theta / 2$. The rotor part of the screw motion is

$$
\begin{aligned}
& \boldsymbol{R} \boldsymbol{L}, \theta=\boldsymbol{\Pi}_{2} \boldsymbol{\Pi}_{1}=\overline{\boldsymbol{n}}+d_{2} \boldsymbol{e}_{\infty} \quad \overline{\boldsymbol{m}}+d_{1} \boldsymbol{e}_{\infty} \\
& =\overline{\boldsymbol{n}} \overline{\boldsymbol{m}}+d_{2} \boldsymbol{e}_{\infty} \overline{\boldsymbol{m}}+d_{1} \overline{\boldsymbol{n}} \boldsymbol{e}_{\infty}
\end{aligned}
$$

There must exist a common point $r$ lying on the intersecting line. The distance from the planes to the origin can be obtained with $d_{2}=\boldsymbol{r} \cdot \overline{\boldsymbol{n}}$ and $d_{1}=\boldsymbol{r} \cdot \overline{\boldsymbol{m}}$. Substituting them into Eq.(10) yields

$$
\begin{aligned}
& \boldsymbol{R} \boldsymbol{L}, \theta=\boldsymbol{R}_{0}+\boldsymbol{e}_{\infty} \boldsymbol{r} \cdot \overline{\boldsymbol{n}} \overline{\boldsymbol{m}}-\boldsymbol{e}_{\infty} \boldsymbol{r} \cdot \overline{\boldsymbol{m}} \overline{\boldsymbol{n}} \\
& =\boldsymbol{R}_{0}+\boldsymbol{e}_{\infty} \boldsymbol{r} \cdot \overline{\boldsymbol{n}} \wedge \overline{\boldsymbol{m}}=\boldsymbol{R}_{0}+\boldsymbol{e}_{\infty} \boldsymbol{r} \underline{\times} \boldsymbol{R}_{0},
\end{aligned}
$$

where $\underline{x}$ is the commutator product with the definition as $\boldsymbol{A} \times \boldsymbol{B}=\frac{1}{2} \boldsymbol{A} \boldsymbol{B}-\boldsymbol{B} \boldsymbol{A}$, more detail can be obtained in Charter 3 of [55]. $\boldsymbol{R}_{0}$ is with the definition in Eq.(5).

The translator along $\overline{\boldsymbol{\omega}}$ with $h \boldsymbol{\theta}$ is

$$
\boldsymbol{T} \overline{\boldsymbol{\omega}}, h \theta=1-\frac{h \theta}{2} \overline{\boldsymbol{\omega}} \boldsymbol{e}_{\infty} .
$$

Thus, the combination of the rotor and the translator can be represented by a motor with the result as

$$
\boldsymbol{M}=\boldsymbol{T} \overline{\boldsymbol{\omega}}, h \boldsymbol{\theta} \quad \boldsymbol{R} \boldsymbol{L}, \theta .
$$

when $h=\infty$, the screw motion becomes a pure translator

$$
\boldsymbol{M}=\boldsymbol{T} \overline{\boldsymbol{\omega}}, \theta
$$

Based on Chasles' theorem, any transformation in the 
space can be represented by a motor as Eq.(13) or Eq.(14).

Besides the screw representation, a motor can also be expressed as

$$
\boldsymbol{M}=\boldsymbol{T} \overline{\boldsymbol{d}}, d \boldsymbol{R}_{0},
$$

which represents a rotation about $\bar{\omega}$ followed with a translation along $\overline{\boldsymbol{d}}$. This form of motor can also represent the arbitrary transformation of geometric entities.

\subsection{Rigid body framing based on the flag in CGA}

A flag is a triple combination of a plane, a line on the plane, and a point on the line, and it can be used to frame a rigid body[58-60]. Hestenes wrote it in CGA form[37] as

$$
\begin{aligned}
& \boldsymbol{F}_{X}=\boldsymbol{P}_{X}+\boldsymbol{L}^{*}+\boldsymbol{\Pi}^{*} \\
& =\boldsymbol{P}_{X}+\boldsymbol{P}_{X} \wedge \boldsymbol{P}_{X 1} \wedge \boldsymbol{e}_{\infty}+\boldsymbol{P}_{X} \wedge \boldsymbol{P}_{X 1} \wedge \boldsymbol{P}_{X 2} \wedge \boldsymbol{e}_{\infty} .
\end{aligned}
$$

It shows that a flag is the sum of 1-blade, 3-blade and 4-blade.

For a better illustration, a flag is represented by a "flag-like" geometry, as shown in Figure 4. The red triangle represents the plane $\boldsymbol{\Pi}^{*}$, the green line represents $\boldsymbol{L}^{*}$ and it is along one of the legs, and the point $\boldsymbol{P}_{X}$ is the vertex of the angle. If a flag is attached on a rigid body, the body is framed, and its position and orientation can be represented by the flag. A special flag $\boldsymbol{F}_{0}$ can be set at the origin, and its plane and line are $\boldsymbol{\Pi}^{*}=\boldsymbol{e}_{0} \wedge \boldsymbol{P}_{01} \wedge \boldsymbol{P}_{02} \wedge \boldsymbol{e}_{\infty} \quad$ and $\quad \boldsymbol{L}^{*}=\boldsymbol{e}_{0} \wedge \boldsymbol{P}_{01} \wedge \boldsymbol{e}_{\infty}$, respectively, where $\boldsymbol{P}_{01}=\mathcal{P}\left(\boldsymbol{e}_{1}\right)$ and $\boldsymbol{P}_{02}=\mathcal{P}\left(\boldsymbol{e}_{2}\right)$. Then this flag is

$$
\boldsymbol{F}_{0}=\boldsymbol{e}_{0}+\boldsymbol{e}_{0} \wedge \boldsymbol{e}_{1} \wedge \boldsymbol{e}_{\infty}+\boldsymbol{e}_{0} \wedge \boldsymbol{e}_{1} \wedge \boldsymbol{e}_{2} \wedge \boldsymbol{e}_{\infty}
$$

Moreover, the triangle of $\boldsymbol{F}_{0}$ is right and its legs are with unit length.

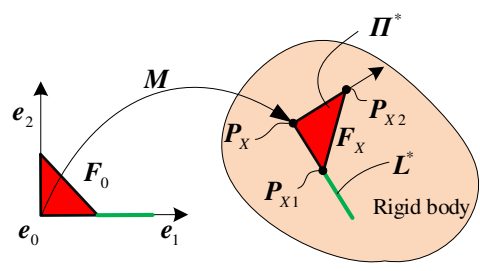

Figure 4 Rigid body framing by flags

A flag and a motor both can reflect a rigid body motion in 3D space. As far as we know, the relationships between the flag and motor have not been studied. It will also show in Section 5.2 that the relationships between them can help us to solve the position kinematics of the parallel mechanism. The relationships are discussed as follows.

\subsubsection{Obtain a flag by a motor}

The flag at origin $\boldsymbol{F}_{o}$ can be moved to the flag $\boldsymbol{F}_{X}$ by a motor $\boldsymbol{M}$, i.e.,

$$
\boldsymbol{F}_{X}=\boldsymbol{M} \boldsymbol{F}_{0} \tilde{\boldsymbol{M}}
$$

What is noteworthy is that $\boldsymbol{F}_{X}$ in Eq.(18) has some limitations besides the form in Eq.(16), for it transformes from $\boldsymbol{F}_{o}$. These limitations are

$$
\left\{\begin{array}{c}
\left\|\mathcal{P}^{-1}\left(\boldsymbol{P}_{X 1}\right)-\mathcal{P}^{-1}\left(\boldsymbol{P}_{X}\right)\right\|=1 \\
\left\|\mathcal{P}^{-1}\left(\boldsymbol{P}_{X 2}\right)-\mathcal{P}^{-1}\left(\boldsymbol{P}_{X}\right)\right\|=1 \\
\mathcal{P}^{-1}\left(\boldsymbol{P}_{X 1}\right)-\mathcal{P}^{-1}\left(\boldsymbol{P}_{X}\right) \cdot \mathcal{P}^{-1}\left(\boldsymbol{P}_{X 2}\right)-\mathcal{P}^{-1}\left(\boldsymbol{P}_{X}\right)=0
\end{array}\right.
$$

which means that these two legs of the flag in Figure 4 are with unit length and orthogonal to each other. The flag is called a standard flag if it satisfies Eq.(19), and called non-standard flag if not. One standard flag $\boldsymbol{F}_{X}$ can be transformed from $\boldsymbol{F}_{0}$ by a motor $\boldsymbol{M}$, as shown in Figure 4.

\subsubsection{Obtain a motor by a flag}

If a standard flag $\boldsymbol{F}_{X}$ can be built directly, the transformation motor that transforms $\boldsymbol{F}_{0}$ to $\boldsymbol{F}_{X}$ satisfies the following equation

$$
\begin{aligned}
& \boldsymbol{F}_{X}=\boldsymbol{M \boldsymbol { F } _ { 0 }} \tilde{\boldsymbol{M}}=\underbrace{\boldsymbol{M} \boldsymbol{e}_{0} \tilde{\boldsymbol{M}}}_{\boldsymbol{P}_{X}}+\boldsymbol{P}_{X} \wedge \underbrace{\boldsymbol{M P _ { 0 1 }} \tilde{\boldsymbol{M}}}_{\boldsymbol{P}_{X 1}} \wedge \boldsymbol{e}_{\infty} \\
& +\boldsymbol{P}_{X} \wedge \boldsymbol{P}_{X 1} \wedge \underbrace{\boldsymbol{M \boldsymbol { P } _ { 0 2 }} \tilde{\boldsymbol{M}}}_{\boldsymbol{P}_{X 2}} \wedge \boldsymbol{e}_{\infty}
\end{aligned}
$$

where $\boldsymbol{M}=\boldsymbol{T} \overline{\boldsymbol{d}}, \boldsymbol{d} \boldsymbol{R}_{0}$. The elements of 1-vector of $\boldsymbol{F}_{X}$ can be expanded as

$$
\left\langle\boldsymbol{F}_{X}\right\rangle_{1}=\boldsymbol{M} \boldsymbol{e}_{0} \tilde{\boldsymbol{M}}=d \overline{\boldsymbol{d}}+\frac{1}{2} d^{2} \boldsymbol{e}_{\infty}+\boldsymbol{e}_{0}
$$

Based on Eq.(21), $d$ and $\overline{\boldsymbol{d}}$ can be obtained, and $\boldsymbol{T} \overline{\boldsymbol{d}}, d$ can be calculated. Then Eq.(20) can be rewritten by the inverse translation with $\tilde{\boldsymbol{T}} \overline{\boldsymbol{d}}, d$ as follows: 


$$
\begin{aligned}
& \tilde{\boldsymbol{T}} \overline{\boldsymbol{d}}, d \boldsymbol{F}_{X} \boldsymbol{T} \overline{\boldsymbol{d}}, d=\boldsymbol{e}_{0}+\boldsymbol{e}_{0} \wedge \boldsymbol{R}_{0} \boldsymbol{P}_{01} \tilde{\boldsymbol{R}}_{0} \wedge \boldsymbol{e}_{\infty}+ \\
& \boldsymbol{e}_{0} \wedge \boldsymbol{R}_{0} \boldsymbol{P}_{01} \tilde{\boldsymbol{R}}_{0} \wedge \boldsymbol{R}_{0} \boldsymbol{P}_{02} \tilde{\boldsymbol{R}}_{0} \wedge \boldsymbol{e}_{\infty}
\end{aligned}
$$

Based on Eq. (5) and Eq.(7), we can set

$$
\boldsymbol{R}_{0}=s+a \boldsymbol{e}_{1,2}+b \boldsymbol{e}_{1,3}+c \boldsymbol{e}_{2,3},
$$

where $s=\cos \left(\frac{\theta}{2}\right), \quad a=-\omega_{3} \sin \left(\frac{\theta}{2}\right), \quad b=\omega_{2} \sin \left(\frac{\theta}{2}\right)$, and $c=-\omega_{1} \sin \left(\frac{\theta}{2}\right)$. Moreover, the quadratic sum of $a$, $b, c$ and $s$ is 1, i.e., $a^{2}+b^{2}+c^{2}+s^{2}=1$. The sum of the elements of 3-vector and 4-vector of $\tilde{\boldsymbol{T}} \overline{\boldsymbol{d}}, d \boldsymbol{F}_{X} \boldsymbol{T} \overline{\boldsymbol{d}}, d \quad$ is

$$
\begin{aligned}
& \left\langle\tilde{\boldsymbol{T}} \overline{\boldsymbol{d}}, d \boldsymbol{F}_{X} \boldsymbol{T} \overline{\boldsymbol{d}}, d\right\rangle_{3,4}=\boldsymbol{e}_{0} \wedge \boldsymbol{R}_{0} \boldsymbol{P}_{01} \tilde{\boldsymbol{R}}_{0} \wedge \boldsymbol{e}_{\infty}+ \\
& \boldsymbol{e}_{0} \wedge \boldsymbol{R}_{0} \boldsymbol{P}_{01} \tilde{\boldsymbol{R}}_{0} \wedge \boldsymbol{R}_{0} \boldsymbol{P}_{02} \tilde{\boldsymbol{R}}_{0} \wedge \boldsymbol{e}_{\infty}= \\
& -a^{2}-b^{2}+c^{2}+s^{2} \boldsymbol{e}_{1,4,5}+-2 a s-2 b c \boldsymbol{e}_{2,4,5}+ \\
& 2 a c-2 b s \boldsymbol{e}_{3,4,5}+-a^{2}+b^{2}+c^{2}-s^{2} \boldsymbol{e}_{1,2,4,5}+ \\
& -2 a b+2 c s \boldsymbol{e}_{1,3,4,5}+-2 a c-2 b s \boldsymbol{e}_{2,3,4,5}
\end{aligned}
$$

It also has the form as follows:

$$
\begin{aligned}
& \left\langle\tilde{\boldsymbol{T}} \overline{\boldsymbol{d}}, d \boldsymbol{F}_{X} \boldsymbol{T} \overline{\boldsymbol{d}}, d\right\rangle_{3,4}=\kappa_{1,4,5} \boldsymbol{e}_{1,4,5}+\kappa_{2,4,5} \boldsymbol{e}_{2,4,5}+ \\
& \kappa_{3,4,5} \boldsymbol{e}_{3,4,5}+\kappa_{1,2,4,5} \boldsymbol{e}_{1,2,4,5}+\kappa_{1,3,4,5} \boldsymbol{e}_{1,3,4,5}+\kappa_{2,3,4,5} \boldsymbol{e}_{2,3,4,5}
\end{aligned}
$$

which can be calculated directly by the expression of $\boldsymbol{F}_{X}$ and $\boldsymbol{T} \overline{\boldsymbol{d}}, d$. Based on the equalities of the terms corresponding to $\boldsymbol{e}_{3,4,5}, \boldsymbol{e}_{2,3,4,5}, \boldsymbol{e}_{1,4,5}$, and $\boldsymbol{e}_{1,2,4,5}$ in Eq.(25) and Eq. (24), the following equations can be obtained

$$
a c=\kappa_{A}, \quad c^{2}-a^{2}=\kappa_{B},
$$

where $\kappa_{A}=\frac{\kappa_{3,4,5}-\kappa_{2,3,4,5}}{4}$ and $\kappa_{B}=\frac{\kappa_{1,4,5}+\kappa_{1,2,4,5}}{2}$. The solutions of Eq.(26) are listed as follows:

1) when $\sqrt{\kappa_{B}^{2}+4 \kappa_{A}^{2}} \neq 0$, solving Eq.(26) yields

$$
c^{2}=\frac{\sqrt{\kappa_{B}^{2}+4 \kappa_{A}^{2}}+\kappa_{B}}{2}, \quad a^{2}=\frac{\sqrt{\kappa_{B}^{2}+4 \kappa_{A}^{2}}-\kappa_{B}}{2} .
$$

If $\kappa_{A} \geq 0, a$ and $c$ have the same sign, otherwise if
$\kappa_{A}<0, a$ and $c$ have different signs. $b$ and $s$ can be obtained based on the equality of the terms corresponding to $\boldsymbol{e}_{2,4,5}$ and $\boldsymbol{e}_{1,3,4,5}$ in Eq.(25) and Eq. (24)

$$
b=\frac{-\kappa_{2,4,5} c-\left(\kappa_{1,3,4,5}\right) a}{2 \sqrt{\kappa_{B}^{2}+4 \kappa_{A}^{2}}}, s=\frac{-\kappa_{2,4,5} a+\left(\kappa_{1,3,4,5}\right) c}{2 \sqrt{\kappa_{B}^{2}+4 \kappa_{A}^{2}}}
$$

It means that two results of the rotor $\boldsymbol{R}_{0}$ can be obtained by Eq.(27), and Eq.(28), and any one of them can transform $\boldsymbol{F}_{0}$ to $\boldsymbol{F}_{X}$.

2) when $\sqrt{\kappa_{B}^{2}+4 \kappa_{A}^{2}}=0$, it will satisfy $a=0, c=0$. On the other hand, according to the terms of $\boldsymbol{e}_{1,4,5}$ and $\boldsymbol{e}_{3,4,5}$ in Eq.(25) and Eq. (24), two equations can be obtained: $s^{2}+b^{2}=\kappa_{1,4,5},-2 b s=\kappa_{3,4,5} . \quad b \quad$ and $s$ can then be calculated as follows:

$$
s^{2}=\frac{\sqrt{\kappa_{1,4,5}^{2}+\kappa_{3,4,5}^{2}}+\kappa_{1,4,5}}{2}, b^{2}=\frac{\sqrt{\kappa_{1,4,5}^{2}+\kappa_{3,4,5}^{2}}-\kappa_{1,4,5}}{2} .
$$

If $\kappa_{3,4,5} \geq 0, b$ and $s$ have different signs, otherwise if $\kappa_{3,4,5}<0, b$ and $s$ have the same sign. It means that two results can be obtained by Eq.(29), and any one of them can transform $\boldsymbol{F}_{0}$ to $\boldsymbol{F}_{X}$.

If a non-standard flag $\boldsymbol{F}_{X}^{\prime}$ is built as $\boldsymbol{F}^{\prime}{ }_{X}=\boldsymbol{P}_{X}+\boldsymbol{L}^{\prime{ }^{*}}+\boldsymbol{\Pi}^{{ }^{*}}$, the standard one can be obtained by the following standardization process. The line part of a flag is $\boldsymbol{L}^{\prime *}=\boldsymbol{P}_{X} \wedge \boldsymbol{P}_{X A} \wedge \boldsymbol{e}_{\infty}$, unlike $\boldsymbol{P}_{X 1}, \boldsymbol{P}_{X A}$ does not satisfy Eq.(19). Suppose $\boldsymbol{P}_{X}=\mathcal{P} \quad x_{1} \boldsymbol{e}_{1}+x_{2} \boldsymbol{e}_{2}+x_{3} \boldsymbol{e}_{3}$ and $\boldsymbol{P}_{X A}=\mathcal{P} \quad a_{1} \boldsymbol{e}_{1}+a_{2} \boldsymbol{e}_{2}+a_{3} \boldsymbol{e}_{3}$, then the line is

$$
\begin{aligned}
& \boldsymbol{L}^{\prime^{*}}=-a_{1} x_{2}+a_{2} x_{1} \boldsymbol{e}_{1,2} \wedge \boldsymbol{e}_{\infty}+-a_{1} x_{3}+a_{3} x_{1} \boldsymbol{e}_{1,3} \wedge \boldsymbol{e}_{\infty}+ \\
& -a_{2} x_{3}+a_{3} x_{2} \boldsymbol{e}_{2,3} \wedge \boldsymbol{e}_{\infty}+a_{1}-x_{1} \boldsymbol{e}_{1} \wedge \boldsymbol{e}_{\infty} \wedge \boldsymbol{e}_{0}+ \\
& a_{2}-x_{2} \boldsymbol{e}_{2} \wedge \boldsymbol{e}_{\infty} \wedge \boldsymbol{e}_{0}+a_{3}-x_{3} \boldsymbol{e}_{3} \wedge \boldsymbol{e}_{\infty} \wedge \boldsymbol{e}_{0}
\end{aligned}
$$

The unit vector from $\boldsymbol{P}_{X}$ to $\boldsymbol{P}_{X A}$ is

$$
\overline{\boldsymbol{m}}_{1}=\frac{a_{1}-x_{1}}{a x} \boldsymbol{e}_{1}+\frac{a_{2}-x_{2}}{a x} \boldsymbol{e}_{2}+\frac{a_{3}-x_{3}}{a x} \boldsymbol{e}_{3},
$$

where $a x=\sqrt{a_{1}-x_{1}{ }^{2}+a_{2}-x_{2}{ }^{2}+a_{3}-x_{3}{ }^{2}}$. The point $\boldsymbol{P}_{X 1}$ that satisfies Eq.(19) can then be obtained by

$$
\boldsymbol{P}_{X 1}=\mathcal{P} \quad x_{1} \boldsymbol{e}_{1}+x_{2} \boldsymbol{e}_{2}+x_{3} \boldsymbol{e}_{3}+\overline{\boldsymbol{m}}_{1}
$$


The OPNS representation of the plane of the non-standard flag $\boldsymbol{F}_{X}{ }_{X}$ is $\boldsymbol{\Pi}^{{ }^{*}}$, then its IPNS representation is $\boldsymbol{\Pi}^{\prime}=-\boldsymbol{\Pi}^{\prime *} \boldsymbol{e}_{1,2,3,4,5}$. The normal vector $\boldsymbol{n}$ of the plane can be obtained by $\boldsymbol{\Pi}$. The unit vector from $\boldsymbol{P}_{X}$ to $\boldsymbol{P}_{X B}$ is

$$
\overline{\boldsymbol{m}}_{2}=\frac{-\overline{\boldsymbol{m}}_{1} \wedge \overline{\boldsymbol{n}} \boldsymbol{e}_{1,2,3}}{\left\|\overline{\boldsymbol{m}}_{1} \wedge \overline{\boldsymbol{n}} \boldsymbol{e}_{1,2,3}\right\|}
$$

Thus, the point satisfying Eq. (19) can be obtained by

$$
\boldsymbol{P}_{X 2}=\mathcal{P} \quad x_{1} \boldsymbol{e}_{1}+x_{2} \boldsymbol{e}_{2}+x_{3} \boldsymbol{e}_{3}+\overline{\boldsymbol{m}}_{2}
$$

Based on the result in Eqs.(32), (34) and (16), the standard flag can be obtained according to $\boldsymbol{P}_{X}, \boldsymbol{P}_{X 1}$, and $\boldsymbol{P}_{X 2}$.

If a geometric entity represented in flag $\boldsymbol{F}_{X}$ is denoted as ${ }^{x} \boldsymbol{G}$, then it can be represented in the origin frame $\boldsymbol{F}_{0}$ as

$$
{ }^{0} \boldsymbol{G}=\boldsymbol{M}^{X} \boldsymbol{G} \tilde{\boldsymbol{M}}
$$

\subsection{Twist and screw velocity}

Spatial velocity (or screw) can be obtained by diff erentiating transform matrix with time[29]. Similarly, by diff erentiating motor with time, the spatial velocity in CGA can also be obtained, more details are in [37].

The motor in Eq.(13) contains $\boldsymbol{R} \boldsymbol{L}, \theta$ and $\boldsymbol{T} \overline{\boldsymbol{\omega}}, h \boldsymbol{\theta}$ which are abbreviated as $\boldsymbol{R}$ and $\boldsymbol{T}$, respectively. The derivative of $\boldsymbol{T}$ with respect to time is

$$
\begin{aligned}
& \dot{\boldsymbol{T}}=-\frac{h \dot{\theta}}{2} \overline{\boldsymbol{\omega}} \boldsymbol{e}_{\infty}=\frac{h \dot{\theta}}{2} \boldsymbol{e}_{\infty} \overline{\boldsymbol{\omega}}\left(\boldsymbol{T}+\frac{h \theta}{2} \overline{\boldsymbol{\omega}} \boldsymbol{e}_{\infty}\right) . \\
& =\frac{1}{2} \dot{\theta} h \boldsymbol{e}_{\infty} \overline{\boldsymbol{\omega}} \boldsymbol{T}
\end{aligned}
$$

The spatial angular velocity can be defined as $\boldsymbol{\Omega}=2 \dot{\boldsymbol{R}}_{0} \tilde{\boldsymbol{R}}_{0}$, i.e, $\dot{\boldsymbol{R}}_{0}=\frac{1}{2} \boldsymbol{\Omega} \boldsymbol{R}_{0}$. Based on Eq.(5), the spatial angular velocity can be calculated

$$
\boldsymbol{\Omega}=\frac{\dot{\theta}}{2} \sin \theta \overline{\boldsymbol{\omega}} \overline{\boldsymbol{\omega}}-1-\dot{\theta} \bar{\omega} \boldsymbol{e}_{1,2,3}=-\dot{\theta} \bar{\omega} \boldsymbol{e}_{1,2,3}
$$

Then, the derivative of $\boldsymbol{R}$ in Eq.(11) with respect to time is

$$
\begin{aligned}
& \dot{\boldsymbol{R}}=\dot{\boldsymbol{R}}_{0}+\boldsymbol{e}_{\infty} \boldsymbol{r} \underline{\times} \dot{\boldsymbol{R}}=\frac{1}{2} \boldsymbol{\Omega R}_{0}+\boldsymbol{e}_{\infty} \boldsymbol{r} \underline{\times} \boldsymbol{\Omega R}_{0} \\
& =\frac{1}{2} \boldsymbol{\Omega}+\boldsymbol{e}_{\infty} \boldsymbol{r} \underline{\times} \boldsymbol{\Omega} \quad \boldsymbol{R}
\end{aligned}
$$

The derivative of $\boldsymbol{M}$ can be obtained

$$
\begin{aligned}
& \dot{\boldsymbol{M}}=\dot{\boldsymbol{T}} \boldsymbol{R}+\boldsymbol{T} \dot{\boldsymbol{R}}=\frac{1}{2} \dot{\theta} h \boldsymbol{e}_{\infty} \overline{\boldsymbol{\omega}} \boldsymbol{T R}+\frac{1}{2} \boldsymbol{T} \boldsymbol{\Omega}+\boldsymbol{e}_{\infty} \boldsymbol{r} \times \boldsymbol{\Omega} \boldsymbol{R} \\
& =\frac{1}{2} \dot{\theta} h \boldsymbol{e}_{\infty} \overline{\boldsymbol{\omega}}+\boldsymbol{T} \boldsymbol{\Omega} \tilde{\boldsymbol{T}}+\boldsymbol{T} \boldsymbol{e}_{\infty} \boldsymbol{r} \times \boldsymbol{\Omega} \tilde{\boldsymbol{T}} \boldsymbol{M}
\end{aligned}
$$

Based on the equation $\boldsymbol{T}=1-\frac{h \theta}{2} \overline{\boldsymbol{\omega}} \boldsymbol{e}_{\infty}$, it will give the property that $\boldsymbol{T} \boldsymbol{\Omega} \tilde{\boldsymbol{T}}=\boldsymbol{\Omega}$ and $\boldsymbol{T} \boldsymbol{e}_{\infty} \boldsymbol{r} \times \boldsymbol{\Omega} \tilde{\boldsymbol{T}}=\boldsymbol{e}_{\infty} \boldsymbol{r} \times \boldsymbol{\Omega}$. Substituting these two properties into Eq.(39) yields

$$
\begin{aligned}
& \dot{\boldsymbol{M}}=\frac{\dot{\theta}}{2} \boldsymbol{e}_{\infty} h \overline{\boldsymbol{\omega}}-\boldsymbol{r} \underline{\times}\left(\bar{\omega} \boldsymbol{e}_{1,2,3}\right)-\bar{\omega} \boldsymbol{e}_{1,2,3} M \\
& =\frac{\dot{\theta}}{2} \boldsymbol{e}_{\infty} h \bar{\omega}-\boldsymbol{r} \wedge \overline{\boldsymbol{\omega}} \boldsymbol{e}_{1,2,3}-\bar{\omega} \boldsymbol{e}_{1,2,3} M
\end{aligned} .
$$

If we set $\boldsymbol{v}=h \overline{\boldsymbol{\omega}}-\boldsymbol{r} \wedge \overline{\boldsymbol{\omega}} \boldsymbol{e}_{1,2,3}$, then the twist can be defined as

$$
\bar{V}=\boldsymbol{e}_{\infty} \boldsymbol{v}-\overline{\boldsymbol{\omega}} \boldsymbol{e}_{1,2,3}
$$

It can also be expanded as

$$
\begin{aligned}
& \overline{\boldsymbol{V}}=v_{1} \boldsymbol{e}_{\infty} \wedge \boldsymbol{e}_{1}+v_{2} \boldsymbol{e}_{\infty} \wedge \boldsymbol{e}_{2}+v_{3} \boldsymbol{e}_{\infty} \wedge \boldsymbol{e}_{3}- \\
& \bar{\omega}_{1} \boldsymbol{e}_{2} \wedge \boldsymbol{e}_{3}+\bar{\omega}_{2} \boldsymbol{e}_{1} \wedge \boldsymbol{e}_{3}-\bar{\omega}_{3} \boldsymbol{e}_{1} \wedge \boldsymbol{e}_{2}
\end{aligned}
$$

Thus, the spatial velocity with the twist $\bar{V}$ is

$$
\boldsymbol{V}=\dot{\theta} \overline{\boldsymbol{V}}
$$

and has the relationships with $\boldsymbol{M}$ as

$$
\dot{\boldsymbol{M}}=\frac{1}{2} \boldsymbol{V M}, \quad \dot{\tilde{M}}=-\frac{1}{2} \tilde{\boldsymbol{M}} \boldsymbol{V} .
$$

According to Eq.(14), when $h=\infty$, the twist with pure translation along $\bar{\omega}$ is

$$
\bar{V}=e_{\infty} \bar{\omega}
$$

There exists a map $\mathcal{S}$ from the twist $\bar{V}$. to the vector in $\mathbb{R}^{6}$, which is 


$$
\$=\mathcal{S} \overline{\boldsymbol{V}}=\omega_{1} \quad \omega_{2} \quad \omega_{3} \quad v_{1} \quad v_{2} \quad v_{2}{ }^{\mathrm{T}} \in \mathbb{R}^{6}
$$

\subsection{Diff erentiate blade with time}

A $k$-blade at time 0 is $\boldsymbol{A}_{\langle k\rangle}$ and it moves to $\boldsymbol{A}_{\langle k\rangle}^{\prime}$ by a motor $\boldsymbol{M}$ at time $t$, which means $\boldsymbol{A}_{\langle k\rangle}^{\prime}=\boldsymbol{M} \boldsymbol{A}_{\langle k\rangle} \tilde{\boldsymbol{M}}$. The differential of $\boldsymbol{A}_{\langle k\rangle}$ is

$$
\dot{\boldsymbol{A}}_{\langle k\rangle}^{\prime}=\dot{\boldsymbol{M}} \boldsymbol{A}_{\langle k\rangle} \tilde{\boldsymbol{M}}+\boldsymbol{M} \boldsymbol{A}_{\langle k\rangle} \dot{\tilde{\boldsymbol{M}}}=\frac{1}{2} \boldsymbol{V} \boldsymbol{A}_{\langle k\rangle}^{\prime}-\boldsymbol{A}_{\langle k\rangle}^{\prime} \boldsymbol{V}=\boldsymbol{V} \times \boldsymbol{A}_{\langle k\rangle}^{\prime}
$$

Thus, the differential of a point $\boldsymbol{P}$ in CGA is

$$
\dot{\boldsymbol{P}}=\boldsymbol{V} \underline{\boldsymbol{P}}=\dot{\boldsymbol{\theta}} \boldsymbol{e}_{\infty} \boldsymbol{v}-\overline{\boldsymbol{\omega}} \boldsymbol{e}_{1,2,3} \underline{\times}\left(\boldsymbol{p}+\frac{1}{2} \boldsymbol{p}^{2} \boldsymbol{e}_{\infty}+\boldsymbol{e}_{0}\right)
$$

It will also generate the result as

$$
\dot{p}=\dot{\theta}-\bar{\omega} \wedge p e_{1,2,3}+v
$$

which is the same as the result in Section 4.2 in [29].

Particularly, the velocity of the point traveling through the origin is

$$
\boldsymbol{V} \times \boldsymbol{e}_{0}=\dot{\theta} \boldsymbol{v}
$$

The higher-order differentials of the point are

$$
\begin{aligned}
& \ddot{\boldsymbol{P}}=\dot{\boldsymbol{V}} \underline{\boldsymbol{P}}+\boldsymbol{V} \times \dot{\boldsymbol{P}}=\dot{\boldsymbol{V}} \underline{\times P}+\boldsymbol{V} \underline{\boldsymbol{V}} \underline{\times} \boldsymbol{P} \\
& \dddot{\boldsymbol{P}}=\ddot{\boldsymbol{V}} \underline{\times P}+2 \dot{\boldsymbol{V}} \underline{\times} \underline{\boldsymbol{V}} \times \boldsymbol{P}+\boldsymbol{V} \underline{\boldsymbol{V}} \underline{\times P}+\boldsymbol{V} \underline{\times} \underline{\boldsymbol{P}}
\end{aligned}
$$

\subsection{Screws in different frames}

As shown in Figure 5, a screw ${ }^{j} \boldsymbol{V}$ and a point ${ }^{j} \boldsymbol{P}$ are represented in $\boldsymbol{F}_{j}$. The differential of the point under the motion of the screw is ${ }^{j} \dot{\boldsymbol{P}}={ }^{j} \boldsymbol{V} \underline{\times}{ }^{j} \boldsymbol{P}$. The same screw and the same point can be also represented in $\boldsymbol{F}_{i}$, and the point velocity represented in $\boldsymbol{F}_{i}$ can be transformed by

$$
\begin{aligned}
& { }^{i} \dot{\boldsymbol{P}}=\boldsymbol{M}_{i, j}{ }^{j} \dot{\boldsymbol{P}} \tilde{\boldsymbol{M}}_{i, j}=\boldsymbol{M}_{i, j}{ }^{j} \boldsymbol{V} \times{ }^{j} \boldsymbol{P} \tilde{\boldsymbol{M}}_{i, j} \\
& =\boldsymbol{M}_{i, j}{ }^{j} \boldsymbol{V} \tilde{\boldsymbol{M}}_{i, j} \underline{\times \boldsymbol{M}_{i, j}{ }^{j} \boldsymbol{P} \tilde{\boldsymbol{M}}_{i, j}}
\end{aligned}
$$

where $\boldsymbol{M}_{i, j}$ is the motor transforming $\boldsymbol{F}_{i}$ to $\boldsymbol{F}_{j}$. It can be seen that the screw can be transformed by the motor as follows:

$$
{ }^{i} \boldsymbol{V}=\boldsymbol{M}_{i, j}{ }^{j} \boldsymbol{V} \tilde{\boldsymbol{M}}_{i, j} .
$$

Actually, If we set $\boldsymbol{M}_{i, j}=\boldsymbol{T}_{i, j} \boldsymbol{R}_{i, j},{ }^{j} \boldsymbol{V}=\boldsymbol{e}_{\infty}{ }^{j} \boldsymbol{v}-{ }^{j} \boldsymbol{\omega} \boldsymbol{e}_{1,2,3}$, and ${ }^{i} \boldsymbol{V}=\boldsymbol{e}_{\infty}{ }^{i} \boldsymbol{v}-{ }^{i} \boldsymbol{\omega} \boldsymbol{e}_{1,2,3}$, where the translate vector of $\boldsymbol{T}_{i, j}$ is $\boldsymbol{d}_{i, j}$, then Eq.(54) can be rewritten as

$$
\begin{aligned}
& \boldsymbol{e}_{\infty}{ }^{i} \boldsymbol{v}-{ }^{i} \boldsymbol{\omega} \boldsymbol{e}_{1,2,3} \\
& =\boldsymbol{e}_{\infty} \boldsymbol{T}_{i, j}{ }^{i} \boldsymbol{v}^{\prime} \tilde{\boldsymbol{T}}_{i, j}-\boldsymbol{T}_{i, j}{ }^{i} \boldsymbol{\omega}^{\prime} \tilde{\boldsymbol{T}}_{i, j} \quad \boldsymbol{d}_{i, j} \boldsymbol{e}_{1,2,3} \wedge \boldsymbol{e}_{\infty}+\boldsymbol{e}_{1,2,3} \\
& =\boldsymbol{e}_{\infty}{ }^{i} \boldsymbol{v}^{\prime}-\boldsymbol{d}_{i, j} \wedge{ }^{i} \boldsymbol{\omega}^{\prime} \boldsymbol{e}_{1,2,3}-{ }^{i} \boldsymbol{\omega}^{\prime} \boldsymbol{e}_{1,2,3}
\end{aligned}
$$

where ${ }^{i} \boldsymbol{v}^{\prime}=\boldsymbol{R}_{i, j}{ }^{j} \boldsymbol{v} \tilde{\boldsymbol{R}}_{i, j}$ and ${ }^{i} \boldsymbol{\omega}^{\prime}=\boldsymbol{R}_{i, j}{ }^{j} \boldsymbol{\omega} \tilde{\boldsymbol{R}}_{i, j}$. Eq. (55) indicates that the results is consistent with the adjoint transformation of screws in p.55, ref.[29].

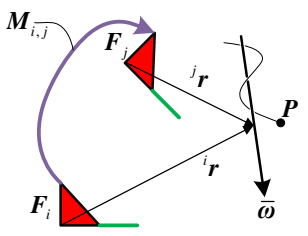

Figure 5 A screw in different frames

\section{Higher-order kinematics of serial manipulators}

\subsection{First-order kinematics}

As shown in Figure 6, two rigid body framed by $\boldsymbol{F}_{i}$ and $\boldsymbol{F}_{j}$ are connected by a joint which can be represented by a twist. At time 0, these two flags have the following relationships:

$$
\boldsymbol{F}_{j}(0)=\boldsymbol{M}_{i, j}^{0} \boldsymbol{F}_{i} \tilde{\boldsymbol{M}}_{i, j}^{0}
$$

At time $t$, the rigid body $\boldsymbol{F}_{j}$ is transformed to $\boldsymbol{F}_{j}(t)$ by the screw motion determined by the joint. The screw motion is represented by the motor $\boldsymbol{M}_{i_{-} j}$ according to Eqs.(13) or (14). Then $\boldsymbol{F}_{j}(t)$ can be calculated by

$$
\boldsymbol{F}_{j}(t)=\boldsymbol{M}_{i_{-} j} \boldsymbol{M}_{i, j}^{0} \boldsymbol{F}_{i} \tilde{\boldsymbol{M}}_{i, j}^{0} \tilde{\boldsymbol{M}}_{i_{-} j}=\boldsymbol{M}_{i_{-} j} \boldsymbol{F}_{j}(0) \tilde{\boldsymbol{M}}_{i_{-} j}
$$

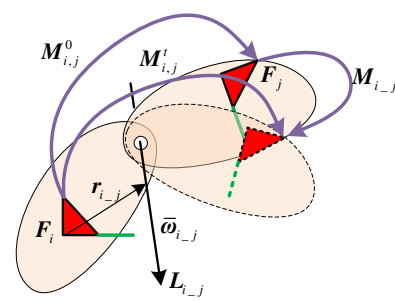

Figure 6 Single joint kinematics 
When $n+1$ rigid bodies (contain the fixed one) are connected by the joints in Figure 7, the flag of the end body $\boldsymbol{F}_{n}(t)$ finally becomes

$\boldsymbol{F}_{n}(t)=\boldsymbol{M}_{0 \_1} \cdots \boldsymbol{M}_{n-2 \_n-1} \boldsymbol{M}_{n-1 \_n} \boldsymbol{F}_{n}(0) \tilde{\boldsymbol{M}}_{n-1 \_n} \tilde{\boldsymbol{M}}_{n-2 \_n-1} \cdots \tilde{\boldsymbol{M}}_{0 \_1}$

The following definitions are given:

$$
\begin{gathered}
\boldsymbol{M}_{i_{-} j}^{t}=\boldsymbol{M}_{i_{-} i+1}^{t} \boldsymbol{M}_{i_{+1} i+2}^{t} \cdots \boldsymbol{M}_{j-1_{-} j}^{t}, \\
\dot{\boldsymbol{M}}_{i_{-} j}^{t}=\frac{1}{2} \boldsymbol{V}_{i_{-} j} \boldsymbol{M}_{i_{-} j}^{t}, \quad 0 \leq i<n, \quad i<j<n,
\end{gathered}
$$

where $\boldsymbol{V}_{i_{-} j}$ is the screw motion of $\boldsymbol{F}_{j}$ relative to the frame $\boldsymbol{F}_{i}$ and it is represented in $\boldsymbol{F}_{i}$. The differential of $\boldsymbol{M}_{0 \_n}^{t} \quad$ can be expanded as

$$
\begin{aligned}
& \dot{\boldsymbol{M}}_{0_{-} n}^{t}=\dot{\boldsymbol{M}}_{0_{-} 1}^{t} \boldsymbol{M}_{1_{-} 2}^{t} \cdots \boldsymbol{M}_{n-1 \_n}^{t}+ \\
& \boldsymbol{M}_{0_{-} 1}^{t} \dot{\boldsymbol{M}}_{1_{-} 2}^{t} \cdots \boldsymbol{M}_{n-1 \_n}^{t}+\cdots \boldsymbol{M}_{0_{-} 1}^{t} \boldsymbol{M}_{1_{-} 2}^{t} \cdots \dot{M}_{n-1_{-} n}^{t}
\end{aligned} .
$$

According to Eq.(59), the velocity of the end rigid body of the serial manipulator can be obtained as

$\boldsymbol{V}_{0_{-} n}=V_{0_{-1}} M_{0_{-} 1}^{t} M_{1_{-} 2}^{t} \cdots M_{n-1 \_n}^{t}+M_{0_{-} 1}^{t} V_{1_{-} 2} M_{1_{-} 2}^{t} \cdots M_{n-1 \_n}^{t}+$

$\cdots \boldsymbol{M}_{0_{-} 1}^{t} \boldsymbol{M}_{1_{2}}^{t} \cdots \dot{\boldsymbol{M}}_{n-1 \_n}^{t} \tilde{\boldsymbol{M}}_{0_{-} n}^{t}$

$=\boldsymbol{V}_{0_{-} 1}+\boldsymbol{M}_{0_{-} 1}^{t} \boldsymbol{V}_{1_{-} 2} \tilde{\boldsymbol{M}}_{0_{-} 1}^{t}+\boldsymbol{M}_{0_{-} 2}^{t} \boldsymbol{V}_{2_{-} 3} \tilde{\boldsymbol{M}}_{0_{-} 2}^{t}+\cdots \boldsymbol{M}_{0_{-} n-1}^{t} \boldsymbol{V}_{n-1_{-} n} \tilde{\boldsymbol{M}}_{0_{-} n-1}^{t}$

If we set ${ }^{0} \boldsymbol{V}_{i_{-} i+1}=\boldsymbol{M}_{0_{-} i}^{t} \boldsymbol{V}_{i_{-} i+1} \tilde{\boldsymbol{M}}_{0_{-} i}^{t}$, i.e., transform $\boldsymbol{V}_{i_{-} i+1}$ to the frame $\boldsymbol{F}_{0}$, then Eq.(61) can be rewritten as

$$
{ }^{0} \boldsymbol{V}_{0_{-} n}={ }^{0} \boldsymbol{V}_{0_{-} 1}+{ }^{0} \boldsymbol{V}_{1_{-} 2}+{ }^{0} \boldsymbol{V}_{2_{-} 3}+\cdots{ }^{0} \boldsymbol{V}_{n-1 \_n} .
$$

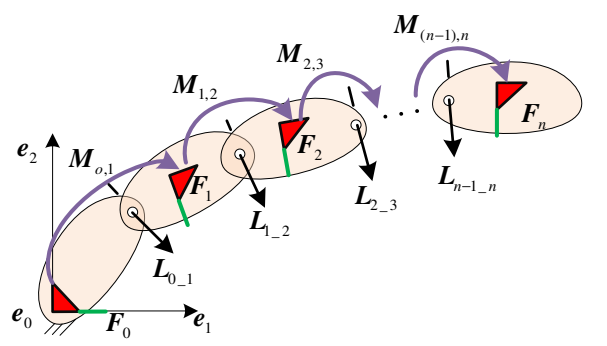

Figure 7 Kinematics of serial manipulator

The above equation can also be written as the linear combination of unit scres, i.e.,

$$
\begin{aligned}
& { }^{0} \boldsymbol{V}_{0_{-} n}=\dot{\theta}_{0_{-} 1}{ }^{0} \overline{\boldsymbol{V}}_{0_{-} 1}+\dot{\theta}_{1_{-} 2}{ }^{0} \overline{\boldsymbol{V}}_{1_{-} 2}+\dot{\theta}_{2_{-} 3}{ }^{0} \overline{\boldsymbol{V}}_{2_{-} 3}+\cdots \dot{\theta}_{n-1 \_n}{ }^{0} \overline{\boldsymbol{V}}_{n-1, n} \\
& =\sum_{i=0}^{n-1} \dot{\theta}_{i_{-} i+1}{ }^{0} \overline{\boldsymbol{V}}_{i_{-} i+1}
\end{aligned}
$$

By considering the mapping ${ }^{0} \overline{\boldsymbol{S}}_{i_{-} i+1}=\mathcal{S}{ }^{0} \overline{\boldsymbol{V}}_{i_{-} i+1}$, the Jacobian matrix can be obtained

$$
{ }^{0} \mathbf{\$}_{0_{-} n}=\mathbf{J}_{0_{-} n} \dot{\boldsymbol{\theta}}_{0_{-} n},
$$

where $\quad \dot{\boldsymbol{\theta}}_{0_{-} n}=\left[\begin{array}{llll}\dot{\theta}_{0 \_1} & \dot{\theta}_{1_{-} 2} & \cdots & \dot{\theta}_{n-1 \_n}\end{array}\right]^{\mathrm{T}} \quad$ and $\mathbf{J}_{0 \_n}=\left[\begin{array}{llll}{ }^{0} \overline{\$}_{0_{-} 1} & { }^{0} \overline{\$}_{1_{-} 2} & \cdots & { }^{0} \overline{\$}_{n-1 \_n}\end{array}\right]$.

The inverse of Eq.(64) is

$$
\dot{\boldsymbol{\theta}}_{0_{-} n}=\mathbf{J}_{0_{-} n}^{ \pm}{ }^{0} \mathbf{S}_{0_{-} n},
$$

where $\mathbf{J}_{0_{-} n}^{ \pm}$is the inverse or the Moore-Penrose inverse of the matrix $\mathbf{J}_{0_{-} n}$.

\subsection{Higher-order kinematics}

Derivate Eq. (63) with respect to time, it follows

$$
{ }^{0} \dot{\boldsymbol{V}}_{0_{-} n}=\sum_{i=1}^{n-1} \ddot{\theta}_{i_{-} i+1}^{0} \overline{\boldsymbol{V}}_{i_{-} i+1}+\sum_{i=1}^{n-1} \dot{\theta}_{i_{-} i+1}{ }^{0} \dot{\overline{\boldsymbol{V}}}_{i_{-} i+1} .
$$

As ${ }^{0} \overline{\boldsymbol{V}}_{i_{-} i+1}$ has relationships with all the joints before it, the derivation of it can be written as

$$
{ }^{0} \dot{\overline{\boldsymbol{V}}}_{i_{-} i+1}={ }^{0} \boldsymbol{V}_{0_{-} i} \times{ }^{0} \overline{\boldsymbol{V}}_{i_{-} i+1},
$$

where ${ }^{0} \boldsymbol{V}_{0_{-} i}=\left(\sum_{j=0}^{i-1} \dot{\theta}_{j_{-} i+1}{ }^{0} \overline{\boldsymbol{V}}_{j_{-} j+1}\right)$. Thus, the acceleration of end body is

$$
{ }^{0} \dot{\boldsymbol{V}}_{0_{-} n}=\sum_{i=1}^{n-1} \ddot{\theta}_{i_{-} i+1}{ }^{0} \overline{\boldsymbol{V}}_{i_{-} i+1}+\sum_{i=1}^{n-1} \dot{\theta}_{i_{-} i+1}{ }^{0} \boldsymbol{V}_{0_{-} i} \times{ }^{0} \overline{\boldsymbol{V}}_{i_{-} i+1}
$$

The above acceleration can also be written as the form of a vector in $\mathbb{R}^{6}$, that is

$$
{ }^{0} \dot{\boldsymbol{\$}}_{0_{-} n}=\mathbf{J}_{0_{-} n} \ddot{\boldsymbol{\theta}}_{0_{-} n}+\mathcal{S}\left(\sum_{i=1}^{n-1} \dot{\theta}_{i_{-} i+1}{ }^{0} \boldsymbol{V}_{0_{-} i} \times{ }^{0} \overline{\boldsymbol{V}}_{i_{-} i+1}\right)
$$

Derivating Eq. (68) with respect to time yields the jerk of end body as follows: 
${ }^{0} \ddot{\boldsymbol{V}}_{0_{-} n}=\sum_{i=1}^{n-1} \dddot{\theta}_{i_{-} i+1}{ }^{0} \overline{\boldsymbol{V}}_{i_{-} i+1}+\sum_{i=1}^{n-1} \ddot{\theta}_{i_{-} i+1}{ }^{0} \dot{\overline{\boldsymbol{V}}}_{i_{-} i+1}+$

$\sum_{i=1}^{n-1} \ddot{\theta}_{i_{-} i+1}{ }^{0} \boldsymbol{V}_{0_{-} i} \times{ }^{0} \overline{\boldsymbol{V}}_{i_{-} i+1}+\sum_{i=1}^{n-1} \dot{\theta}_{i_{-} i+1}\left(\frac{d^{0} \boldsymbol{V}_{0_{-} i} \underline{x}^{0} \overline{\boldsymbol{V}}_{i_{-} i+1}}{d t}\right)$,

$=\sum_{i=1}^{n-1} \dddot{\theta}_{i_{-} i+1}{ }^{0} \overline{\boldsymbol{V}}_{i_{-} i+1}+2 \sum_{i=1}^{n-1} \ddot{\theta}_{i_{-} i+1}{ }^{0} \boldsymbol{V}_{0_{-} i} \times{ }^{0} \overline{\boldsymbol{V}}_{i_{-} i+1}+$

$\sum_{i=1}^{n-1} \dot{\theta}_{i_{-} i+1}\left(\dot{V}_{0_{-} i} \times{ }^{0} \overline{\boldsymbol{V}}_{i_{-} i+1}+{ }^{0} \boldsymbol{V}_{0_{-} i} \times\left(\bar{V}_{0_{-} i} \times{ }^{0} \overline{\boldsymbol{V}}_{i_{-} i+1}\right)\right.$

where ${ }^{0} \dot{\boldsymbol{V}}_{0_{-} i}=\sum_{j=1}^{i-1} \ddot{\theta}_{j_{-} j+1}{ }^{0} \overline{\boldsymbol{V}}_{j_{-} j+1}+\sum_{j=1}^{n-1} \dot{\theta}_{i_{-} i+1}{ }^{0} \boldsymbol{V}_{0_{-} j} \underline{\times}^{0} \overline{\boldsymbol{V}}_{j_{-} j+1}$.

For programming convenience, the iteration algorithms of velocity, acceleration, and jerk are given

$$
\begin{gathered}
{ }^{0} \boldsymbol{V}_{0_{-} k}={ }^{0} \boldsymbol{V}_{0_{-} k-1}+\dot{\boldsymbol{\theta}}_{k-1 \_k}{ }^{0} \overline{\boldsymbol{V}}_{k-1 \_k}, \\
{ }^{0} \dot{\boldsymbol{V}}_{0_{-} k}={ }^{0} \dot{\boldsymbol{V}}_{0_{-} k-1}+\ddot{\boldsymbol{\theta}}_{k-1 \_k}{ }^{0} \overline{\boldsymbol{V}}_{k-1 \_k}+\dot{\boldsymbol{\theta}}_{k-1 \_k}{ }^{0} \boldsymbol{V}_{0_{-} k-1} \underline{ }^{0} \overline{\boldsymbol{V}}_{k-1 \_k},
\end{gathered}
$$

${ }^{0} \ddot{\boldsymbol{V}}_{0_{-} k}={ }^{0} \ddot{\boldsymbol{V}}_{0_{-} k-1}+\dddot{\boldsymbol{\theta}}_{k-1 \_k}{ }^{0} \overline{\boldsymbol{V}}_{k-1 \_k}+2 \ddot{\boldsymbol{\theta}}_{k-1 \_k}{ }^{0} \boldsymbol{V}_{0_{-} k-1} \underline{ }^{0} \overline{\boldsymbol{V}}_{k-1 \_k}+$

$\dot{\theta}_{k-1 \_k}{ }^{0} \dot{\boldsymbol{V}}_{0_{-} k-1} \underline{ }^{0} \overline{\boldsymbol{V}}_{k-1 \_k}+{ }^{0} \boldsymbol{V}_{0_{-} k-1} \underline{{ }^{0} \boldsymbol{V}_{0_{-} k-1}} \underline{ }^{0} \overline{\boldsymbol{V}}_{k-1, k}$

\section{Higher-order kinematics of the 3-RRS parallel mechanism}

Kinematics of parallel mechanism can be obtained based on the methods with serial manipulator. For the lower-mobility 3-RRS mechanism, the six-dimentional motions of the output platform are constrained by the structure. The calculation of the Motion constraints is the key for the inverse kinematics of it.

\subsection{Motion constraints of the output platform}

As shown in Figure 8, the 3-RRS mechanism is constructed by three symmetrical RRS ( $\mathrm{R}$ and $\mathrm{S}$ represent revolute and Spherical joints, respectively) limbs, i.e., the first and the second joints are paralleled revolute joints, and the third joint is a spherical joint which can be treated as three independent revolute joints intersecting in one point. The center of the joints $\mathrm{R}, \mathrm{R}$, and $\mathrm{S}$ are $A_{i}, B_{i}$, and $C_{i}$, respectively, where $i=1,2,3$. These three limbs are distributed in three planes which are all perpendicular to the down plane determined by the equilateral triangle $A_{1} A_{2} A_{3}$, whose center is the origin $O$. The output platform of the mechanism connects with the three limbs by the spherical joints which also distribute as an equilateral triangle $C_{1} C_{2} C_{3}$ with a center $H$. The lengths of $A_{i} O$ and $C_{i} H$ are both $r$, and the lengths of $A_{i} B_{i}$ and $B_{i} C_{i}$ are both $l$, as shown in Figure 9. In each limb, a linear actuator is distributed by connecting $E_{i}$ and $F_{i}$, which are attached on the base and the link $B_{i} C_{i}$ respectively. The distance between $E_{i}$ and $F_{i}$ is $q_{i}$ which is seen as the input of the mechanism. The dimensions of the mechanism are listed in Table 2.

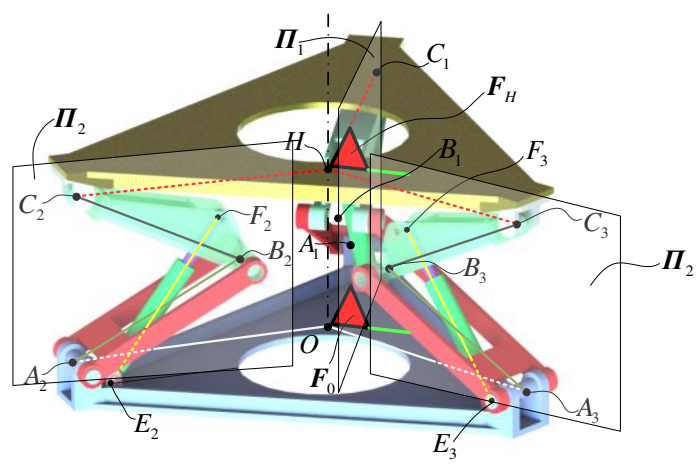

Figure 8 The 3-RRS mechanism

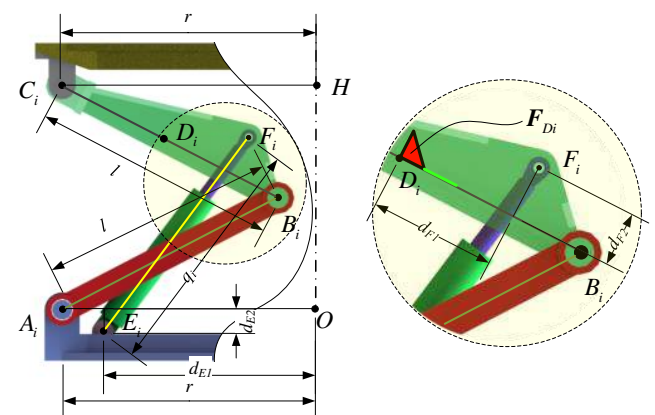

Figure 9 The geometry parameters of the $i$-th limb and actuator

Table 2 Dimensions of the 3-RRS mechanism

\begin{tabular}{cccccc}
\hline$r(\mathrm{~m})$ & $l(\mathrm{~m})$ & $d_{E 1}(\mathrm{~m})$ & $\begin{array}{l}d_{E 2} \\
(\mathrm{~m})\end{array}$ & $d_{F 1}(\mathrm{~m})$ & $\begin{array}{l}d_{F 2} \\
(\mathrm{~m})\end{array}$ \\
\hline 1.2 & 0.9 & 1.05 & 0.08 & 0.25 & 0.15 \\
\hline
\end{tabular}

The flag at the origin is located in the center of the triangle $A_{1} A_{2} A_{3}$ and is denoted as $\boldsymbol{F}_{0}$. The flag of the output platform is fixed at $H$, and is denoted as $\boldsymbol{F}_{H}$. The motor from $\boldsymbol{F}_{0}$ to $\boldsymbol{F}_{H}$ is

$$
\boldsymbol{M}_{0, H}=\boldsymbol{T}_{0, H} \boldsymbol{R}_{3} \alpha \boldsymbol{R}_{2} \quad \beta \quad \boldsymbol{R}_{1} \gamma,
$$

where $\boldsymbol{T}_{0, H}=1-x \boldsymbol{e}_{1}+y \boldsymbol{e}_{2}+z \boldsymbol{e}_{3} \boldsymbol{e}_{\infty}$. According to Eq.(74), $\boldsymbol{F}_{H}$ can be transformed from $\boldsymbol{F}_{0}$ by the following elementary rotations and translations:

- Rotate $\boldsymbol{F}_{0}$ by the angle $\gamma$ about $\boldsymbol{e}_{1}$ of $\boldsymbol{F}_{0}$ to the first flag; 
1) Rotate the first flag by the angle $\beta$ about $\boldsymbol{e}_{2}$ of $\boldsymbol{F}_{0}$ to the second flag;

2) Rotate the second flag by the angle $\alpha$ about $\boldsymbol{e}_{3}$ of $\boldsymbol{F}_{0}$ to the third flag;

3) Translate the third flag by the vector $x \boldsymbol{e}_{1}+y \boldsymbol{e}_{2}+z \boldsymbol{e}_{3}$ to the final flag $\boldsymbol{F}_{H}$.

However, according to the DoF of 3-RRS, the above rotations and translation are not free. If the motion parameter $z, \beta$ and $\gamma$ are set to be free, $x, y$, and $\alpha$ can be determined by them. The points $A_{1}, A_{2}$, and $A_{3}$ represented in $\boldsymbol{F}_{0}$ are

$$
\begin{aligned}
\boldsymbol{P}_{A 1} & =\mathcal{P} r \boldsymbol{e}_{2}, \\
\boldsymbol{P}_{A 2} & =\mathcal{P}\left(-\frac{\sqrt{3}}{2} r \boldsymbol{e}_{1}-\frac{r}{2} \boldsymbol{e}_{2}\right), \\
\boldsymbol{P}_{A 3} & =\mathcal{P}\left(\frac{\sqrt{3}}{2} r \boldsymbol{e}_{1}-\frac{r}{2} \boldsymbol{e}_{2}\right),
\end{aligned}
$$

respectively. As the joints' distribution of the output platform is the same as the fixed platform determined by $A_{1} A_{2} A_{3}$, the points $C_{1}, C_{2}$, and $C_{3}$ represented in $\boldsymbol{F}_{H}$ is the same as the equations in Eq.(75), respectively. Then the points $C_{1}, C_{2}$, and $C_{3}$ represented in $\boldsymbol{F}_{0}$ can be obtained as follows

$$
\begin{gathered}
\boldsymbol{P}_{C 1}=\boldsymbol{M}_{0, H} \boldsymbol{P}_{A 1} \tilde{\boldsymbol{M}}_{0, H}, \\
\boldsymbol{P}_{C 2}=\boldsymbol{M}_{0, H} \boldsymbol{P}_{A 2} \tilde{\boldsymbol{M}}_{0, H}, \\
\boldsymbol{P}_{C 3}=\boldsymbol{M}_{0, H} \boldsymbol{P}_{A 3} \tilde{\boldsymbol{M}}_{0, H},
\end{gathered}
$$

respectively. Besides, the IPNS representations of the planes $\boldsymbol{\Pi}_{1}, \boldsymbol{\Pi}_{2}$, and $\boldsymbol{\Pi}_{3}$ are

$$
\begin{aligned}
\boldsymbol{\Pi}_{1} & =\boldsymbol{e}_{1}, \\
\boldsymbol{\Pi}_{2} & =-\frac{1}{2} \boldsymbol{e}_{1}+\frac{\sqrt{3}}{2} \boldsymbol{e}_{2}, \\
\boldsymbol{\Pi}_{3} & =-\frac{1}{2} \boldsymbol{e}_{1}-\frac{\sqrt{3}}{2} \boldsymbol{e}_{2},
\end{aligned}
$$

respectively. The constraint of the output platform is that the points $C_{1}, C_{2}$, and $C_{3}$ must lie on $\boldsymbol{\Pi}_{1}, \boldsymbol{\Pi}_{2}$, and $\boldsymbol{\Pi}_{3}$, respectively, i.e.

$$
\boldsymbol{P}_{C 1} \cdot \boldsymbol{\Pi}_{1}=0, \quad \boldsymbol{P}_{C 2} \cdot \boldsymbol{\Pi}_{2}=0 \text {, and } \boldsymbol{P}_{C 3} \cdot \boldsymbol{\Pi}_{3}=0 \text {. }
$$

By solving the above equations, $x, y$, and $\alpha$ can be expressed by $z, \beta$, and $\gamma$ as follows:

1) When $\beta \neq 0$ or $\gamma \neq 0$, the results are

$$
\alpha=\arctan \left(\frac{\mathrm{c} \beta \mathrm{c} \gamma-1 \mathrm{~s} \gamma \mathrm{s} \beta}{\mathrm{c} \beta^{2} \mathrm{c} \gamma^{2}-1}, \frac{\mathrm{c} \gamma+\mathrm{c} \beta \mathrm{c} \beta \mathrm{c} \gamma-1}{\mathrm{c} \beta^{2} \mathrm{c} \gamma^{2}-1}\right),
$$

$$
\begin{gathered}
x=-\frac{\frac{r \mathrm{~s} \beta \mathrm{s} \gamma \mathrm{c} \beta \mathrm{c} \gamma-1 \mathrm{c} \beta}{\mathrm{c} \beta^{2} \mathrm{c} \gamma^{2}-1}}{\sqrt{\frac{\mathrm{s} \beta^{2} \mathrm{~s} \gamma^{2}+\mathrm{c} \beta^{2}+2 \mathrm{c} \beta \mathrm{c} \gamma+\mathrm{c} \gamma^{2}}{\mathrm{c} \beta^{2} \mathrm{c} \gamma^{2}+2 \mathrm{c} \beta \mathrm{c} \gamma+1}}} \\
y=\frac{1}{2} \frac{\mathrm{c} \beta^{2} \mathrm{c} \gamma^{2}-2 \mathrm{c} \beta^{2}+1 r \mathrm{c} \beta \mathrm{c} \gamma-1}{\mathrm{c} \beta^{2} \mathrm{c} \gamma^{2}-1} .
\end{gathered}
$$

2) When $\beta=0$ and $\gamma=0$, the result are $\alpha=0$, $x=0, y=0$.

If the motion laws of $z, \beta$ and $\gamma$ are given in Table 3, the six DoF motions of the output platform can be obtained and drawn in Figure 10 in the period of $10 \mathrm{~s}$. The trace of the flag $\boldsymbol{F}_{H}$ is also given in Figure 11. It can be seen that the flag $\boldsymbol{F}_{H}$ can represent the motions of the output platform.

Table 3 The motion laws of the output platform

\begin{tabular}{ccc}
\hline$z(\mathrm{~m})$ & $\beta(\mathrm{rad})$ & $\gamma(\mathrm{rad})$ \\
\hline $1+0.2 \sin \left(\frac{2 \pi}{10} t\right)$ & $\frac{25}{180} \pi \sin \left(\frac{2 \pi}{8} t\right)$ & $\frac{20}{180} \pi \sin \left(\frac{2 \pi}{5} t\right)$ \\
\hline
\end{tabular}

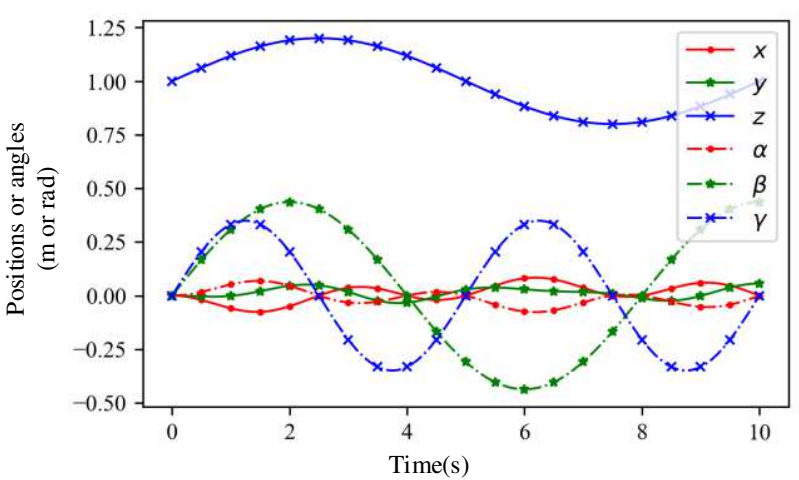

Figure 10 Six DoF motions of the output platform 


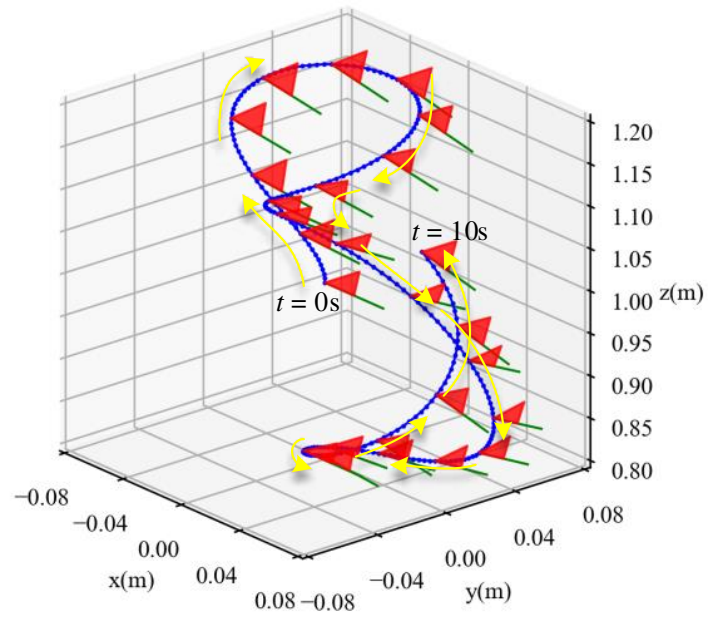

Figure 11 The trace of the flag attatched on the output platform from $0 \mathrm{~s}$ to $10 \mathrm{~s}$

Taking the differential of Eq.(74) yields

$\dot{\boldsymbol{M}}_{0, H}=\dot{\boldsymbol{T}}_{0, H} \boldsymbol{R}_{z} \alpha \boldsymbol{R}_{y} \quad \beta \quad \boldsymbol{R}_{x} \gamma+\boldsymbol{T}_{0, H} \dot{\boldsymbol{R}}_{z} \alpha \boldsymbol{R}_{y} \quad \beta \quad \boldsymbol{R}_{x} \gamma+$ $\begin{array}{llllllllllllll}\boldsymbol{T}_{0, H} \boldsymbol{R}_{z} & \alpha & \dot{\boldsymbol{R}}_{y} & \beta & \boldsymbol{R}_{x} & \gamma+\boldsymbol{T}_{0, H} \boldsymbol{R}_{z} & \alpha & \boldsymbol{R}_{y} & \beta & \dot{\boldsymbol{R}}_{x} & \gamma\end{array}$

$=\frac{1}{2} \boldsymbol{V}_{0_{-} H} \boldsymbol{M}_{0, H}$

The velocity $\boldsymbol{V}_{0 \_H}$ of the output platform can be calculated by Eq.(82), and its results are given in Eq.(104) from Appendix.

As the motions of the three points $C_{1}, C_{2}$, and $C_{3}$ are restricted on the planes $\boldsymbol{\Pi}_{1}, \boldsymbol{\Pi}_{2}$, and $\boldsymbol{\Pi}_{3}$, respectively, the velocities of the points should always be perpendicular to the normal vector of these planes

$$
\begin{gathered}
\boldsymbol{V}_{0_{-} H} \underline{\times} \boldsymbol{P}_{C 1} \cdot \boldsymbol{\Pi}_{1}=0, \\
\boldsymbol{V}_{0_{-} H} \underline{\times} \boldsymbol{P}_{C 2} \cdot \boldsymbol{\Pi}_{2}=0, \\
\boldsymbol{V}_{0_{-} H} \times \boldsymbol{P}_{C 3} \cdot \boldsymbol{\Pi}_{3}=0 .
\end{gathered}
$$

The solutions of the above equations are

$$
\dot{x}=\frac{r A_{x}}{2 B_{x}}, \quad \dot{y}=\frac{r}{4} \frac{A_{y}}{B_{x}}, \text { and } \dot{\alpha}=-\frac{A_{\alpha}}{B_{x}},
$$

where the expressions of $A_{x}, A_{y}, A_{\alpha}$, and $B_{x}$ are listed in Appendix. Based on the motion laws in Table 3, the velocity of the output platform are calculated and illustrated in Figure 12.

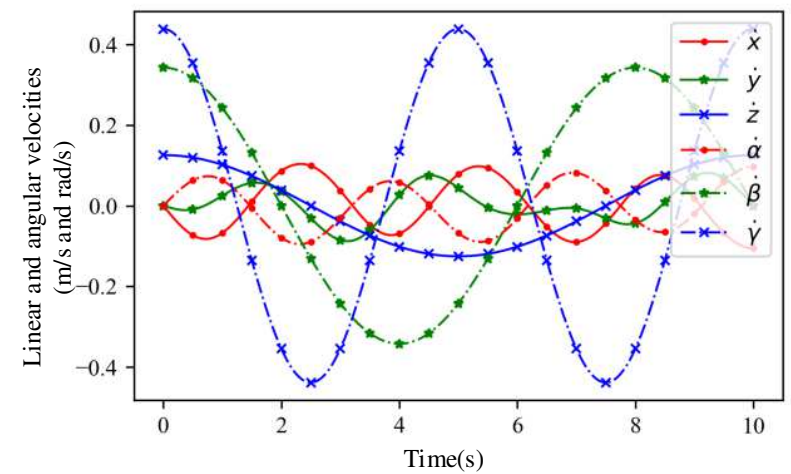

Figure 12 Velocity of the output platform

By the multi-derivations of Eq.(104), the acceleration $\dot{\boldsymbol{V}}_{0_{-} H}$ and the jerk $\ddot{\boldsymbol{V}}_{\mathrm{O}_{-} H}$ of the output platform can be obtained. By the multi derivations of Eq.(84), the constraint of acceleration and jerk can also be obtained.

\subsection{Higher-order kinematics of 3-RRS}

The kinematics of the 3-RRS mechanism is about the methods to obtain the positions, velocities, accelerations, and jerks of the actuators based on the motion of the output platform.

\subsubsection{Calculate the length of the actuators}

Based on $\boldsymbol{M}_{0, H}$ and Eq. (76), the points $\boldsymbol{P}_{C 1}, \boldsymbol{P}_{C 2}$, and $\boldsymbol{P}_{C 3}$ represented in the flag $\boldsymbol{F}_{0}$ can be obtained. Then, the sphere, whose center is $\boldsymbol{P}_{C i}(i=1,2,3)$ and radius is $l$, is expressed by IPNS as

$$
\boldsymbol{S}_{C i}=\boldsymbol{P}_{C i}-\frac{1}{2} l^{2} \boldsymbol{e}_{\infty} .
$$

Besides, the sphere, whose center is $\boldsymbol{P}_{A i}(i=1,2,3)$ and radius is $l$, is expressed by IPNS as

$$
\boldsymbol{S}_{A i}=\boldsymbol{P}_{A i}-\frac{1}{2} l^{2} \boldsymbol{e}_{\infty}
$$

The intersection of $\boldsymbol{S}_{A i}, \boldsymbol{S}_{C i}$, and $\boldsymbol{\Pi}_{i}$ is point pair, which can be expressed as

$$
\boldsymbol{P p}_{i}=\boldsymbol{S}_{A i} \wedge \boldsymbol{S}_{C i} \wedge \boldsymbol{\Pi}_{i}
$$

Based on $\boldsymbol{P} \boldsymbol{p}_{i}$, the point $B_{i}$ can be obtained by

$$
\boldsymbol{P}_{B i}=\frac{ \pm \sqrt{\boldsymbol{P p}_{i}^{*} \cdot \boldsymbol{P} \boldsymbol{p}_{i}^{*}}+\boldsymbol{P p _ { i }}{ }^{*}}{\boldsymbol{e}_{\infty} \cdot \boldsymbol{P p}_{i}{ }^{*}}
$$

where $\boldsymbol{\Pi}_{i}^{*}$ is the OPNS representation of $\boldsymbol{\Pi}_{i}$ in 
Eq.(77). $\quad \boldsymbol{P p}_{i}^{*}=-\boldsymbol{P} \boldsymbol{p}_{i} \boldsymbol{e}_{1,2,3,4,5}$. Eq.(88) provides two results of $\boldsymbol{P}_{B i}$, and the one which is closer to the origin is selected.

The flag $\boldsymbol{F}_{D i}$ attached on the link $B_{i} C_{i}$ is built at the midpoint $D_{i}$ of $B_{i} C_{i}$. The point can be calculated by

$$
\boldsymbol{P}_{D i}=\mathcal{P}\left(\frac{\mathcal{P}^{-1} \boldsymbol{P}_{B i}+\mathcal{P}^{-1} \boldsymbol{P}_{C i}}{2}\right) .
$$

According to Figure 9, the flag $\boldsymbol{F}_{D i}$ represented in $\boldsymbol{F}_{0}$ is

$$
{ }^{0} \boldsymbol{F}_{D i}=\boldsymbol{P}_{D i}+\boldsymbol{P}_{D i} \wedge \boldsymbol{P}_{B i} \wedge \boldsymbol{e}_{\infty}+\boldsymbol{\Pi}_{i}^{*},
$$

where the standard form of ${ }^{0} \boldsymbol{F}_{D i}$ can be obtained based on Eqs. (30) (34). Moreover, the motor $\boldsymbol{M}_{0_{-} D i}$ from $\boldsymbol{F}_{0}$ to $\boldsymbol{F}_{D i}$ can be calculated by Eq (21), and Eqs. (27) $\sim(29)$. The point $F_{i}$, which is on the head of the actuators, can be represented in $\boldsymbol{F}_{D i}$ as

$$
{ }^{D i} \boldsymbol{P}_{F i}=\mathcal{P} d_{F 1} \boldsymbol{e}_{1}+d_{F 2} \boldsymbol{e}_{2},
$$

and it can be transformed into $\boldsymbol{F}_{0}$ by

$$
\boldsymbol{P}_{F i}=\boldsymbol{M}_{0_{-} D i}{ }^{D i} \boldsymbol{P}_{F i} \tilde{\boldsymbol{M}}_{0_{-} D i} .
$$

Moreover, the fixed points $E_{i}$ represented in $\boldsymbol{F}_{0}$ are

$$
\begin{gathered}
\boldsymbol{P}_{E 1}=\mathrm{P} d_{E 1} \boldsymbol{e}_{2}-d_{E 2} \boldsymbol{e}_{3}, \\
\boldsymbol{P}_{E 2}=\boldsymbol{R}_{z}\left(\frac{2 \pi}{3}\right) \boldsymbol{P}_{E 1} \tilde{\boldsymbol{R}}_{z}\left(\frac{2 \pi}{3}\right), \\
\boldsymbol{P}_{E 3}=\boldsymbol{R}_{z}\left(\frac{2 \pi}{3}\right) \boldsymbol{P}_{E 2} \tilde{\boldsymbol{R}}_{z}\left(\frac{2 \pi}{3}\right)
\end{gathered}
$$

respectively. Then the distance between the point $\boldsymbol{P}_{F i}$ and $\boldsymbol{P}_{E i}$ can be obtained:

$$
q_{i}=\sqrt{-2 \boldsymbol{P}_{F i} \cdot \boldsymbol{P}_{E i}} .
$$

The points and flags of the mechanism at different configurations are drawn in Figure 13, which can indicate the correctness of the calculation methods above.

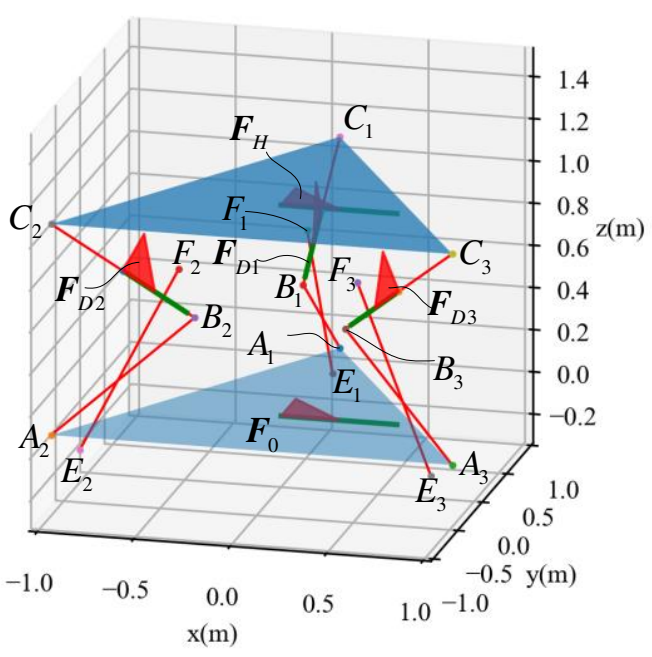

a) $z=1 \mathrm{~m}, \quad \beta=0 \mathrm{deg}$, and $\gamma=0 \mathrm{deg}$

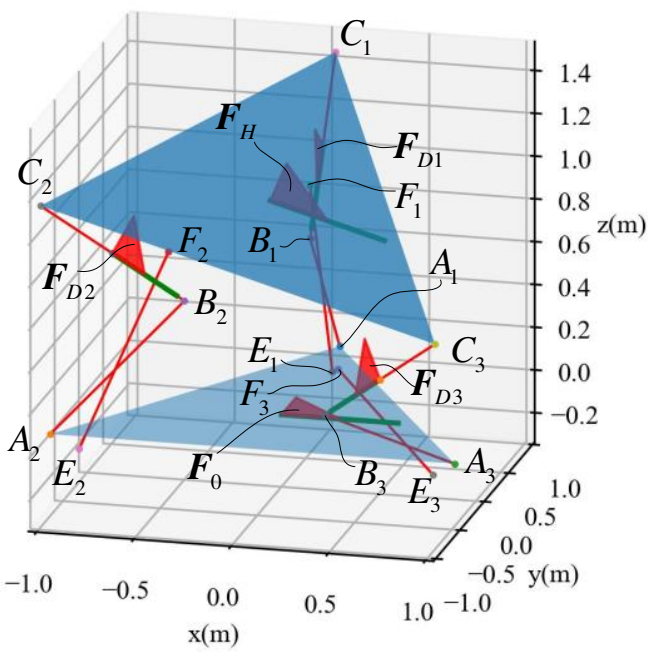

b) $z=1 \mathrm{~m}, \quad \beta=15 \mathrm{deg}$, and $\gamma=20 \mathrm{deg}$

Figure 13 Two configurations of the mechanism with points and flags

\subsubsection{Calculate the velocity of the actuators}

The twist of the $k$-th rigid body of the $i$-th limb represented in $\boldsymbol{F}_{0}$ is donated as ${ }^{0} \boldsymbol{V}_{j_{-} k, i}$, and they can be constructed as follows:

$$
\begin{aligned}
& { }^{0} \overline{\boldsymbol{V}}_{0_{1} 1, i}=\boldsymbol{e}_{\infty} \mathcal{P}^{-1} \boldsymbol{P}_{A i} \wedge \boldsymbol{\Pi}_{i} \boldsymbol{e}_{1,2,3}-\boldsymbol{\Pi}_{i} \boldsymbol{e}_{1,2,3} \\
& { }^{0} \overline{\boldsymbol{V}}_{1_{1} 2, i}=\boldsymbol{e}_{\infty} \mathcal{P}^{-1} \boldsymbol{P}_{B i} \wedge \boldsymbol{\Pi}_{i} \boldsymbol{e}_{1,2,3}-\boldsymbol{\Pi}_{i} \boldsymbol{e}_{1,2,3}, \\
& { }^{0} \overline{\boldsymbol{V}}_{2_{3}, i}=\boldsymbol{e}_{\infty} \mathcal{P}^{-1} \boldsymbol{P}_{C i} \wedge \boldsymbol{e}_{1} \boldsymbol{e}_{1,2,3}-\boldsymbol{e}_{1} \boldsymbol{e}_{1,2,3}, \\
& { }^{0} \overline{\boldsymbol{V}}_{3_{-4, i}}=\boldsymbol{e}_{\infty} \mathcal{P}^{-1} \boldsymbol{P}_{C i} \wedge \boldsymbol{e}_{2} \boldsymbol{e}_{1,2,3}-\boldsymbol{e}_{2} \boldsymbol{e}_{1,2,3}, \\
& { }^{0} \overline{\boldsymbol{V}}_{4_{-} 5, i}=\boldsymbol{e}_{\infty} \mathcal{P}^{-1} \boldsymbol{P}_{C i} \wedge \boldsymbol{e}_{3} \boldsymbol{e}_{1,2,3}-\boldsymbol{e}_{3} \boldsymbol{e}_{1,2,3},
\end{aligned}
$$


where ${ }^{0} \overline{\boldsymbol{V}}_{0_{-} 1, i}$ and ${ }^{0} \overline{\boldsymbol{V}}_{1_{-} 2, i}$ are corresponding to the first and second revolute joints, besides, ${ }^{0} \overline{\boldsymbol{V}}_{2_{-} 3, i},{ }^{0} \overline{\boldsymbol{V}}_{3_{-} 4, i}$, and ${ }^{0} \overline{\boldsymbol{V}}_{4 \_5, i}$ are corresponding to the three revolute axes of the spherical joint. As these axes of the spherical joint is arbitrary, their directions can be set as $\boldsymbol{e}_{1}, \boldsymbol{e}_{2}$, and $\boldsymbol{e}_{3}$ for simplicity. The Jacobian matrix of the $i$-th limb can then be obtained as

$$
\dot{\boldsymbol{\theta}}_{0 \_5, i}=\mathbf{J}_{0 \_}^{ \pm}{ }_{-i} \mathcal{S}{ }^{0} \overline{\boldsymbol{V}}_{0_{-} H}
$$

According to Eq. (71), the velocity of each rigid body $\boldsymbol{V}_{0 \_k, i}$ can be obtained.

By calculating the differential of Eq. (94) and considering $\dot{\boldsymbol{P}}_{F i}=\boldsymbol{V}_{0 \_2, i} \underline{\times \boldsymbol{P}_{F i}}$, the actuator's velocity of each limb can be obtained

$$
\dot{q}_{i}=-\frac{\boldsymbol{V}_{0_{-2, i}} \underline{\times} \boldsymbol{P}_{F i} \cdot \boldsymbol{P}_{E i}}{q_{i}} .
$$

5.2.3 Calculate the acceleration and jerk of the actuators According to Eq. (69), the angular accelerations of the joints on each limb can be obtained as follows:

$$
\ddot{\boldsymbol{\theta}}_{0_{-} 5, i}=\mathbf{J}_{0_{-} \leq 5, i}^{ \pm} \mathcal{S}\left({ }^{0} \dot{\overline{\boldsymbol{V}}}_{0_{-} H}-\sum_{i=1}^{4} \dot{\boldsymbol{\theta}}_{i_{-} i+1}{ }^{0} \boldsymbol{V}_{0_{-} i} \underline{ }^{0} \overline{\boldsymbol{V}}_{i_{-} i+1}\right)
$$

The acceleration $\dot{\boldsymbol{V}}_{0_{-} k, i}$ of each rigid body can then be calculated based on the angular velocities and accelerations. Derivate Eq. (97) with respect to time, it follows

$$
\ddot{q}_{i}=\frac{-\dot{q}_{i}^{2}-\ddot{\boldsymbol{P}}_{F i} \cdot \boldsymbol{P}_{E i}}{q_{i}}
$$

where $\ddot{\boldsymbol{P}}_{F i}=\dot{\boldsymbol{V}}_{0 \_2, i} \underline{\times \boldsymbol{P}_{F i}}+\boldsymbol{V}_{0 \_2, i} \underline{\times} \boldsymbol{V}_{0_{-} 2, i} \underline{\times \boldsymbol{P}_{F i}} \quad$ according to Eq.(51).

According to Eq. (70), the angular jerk of the joints on each limb can be obtained by Eq.(105) in Appendix. Derivate Eq. (99) with respect to time, it follows

$$
\dddot{q}_{i}=\frac{-3 \dot{q}_{i} \ddot{q}_{i}-\dddot{\boldsymbol{P}}_{F i} \cdot \boldsymbol{P}_{E i}}{q_{i}},
$$

where $\dddot{\boldsymbol{P}}_{F i}=\ddot{\boldsymbol{V}}_{0 \_2, i} \times \boldsymbol{P}_{F i}+2 \dot{\boldsymbol{V}}_{0_{-} 2, i} \underline{\boldsymbol{V}_{0 \_} 2, i} \times \boldsymbol{P}_{F i}+$ $\boldsymbol{V}_{0 \_2, i} \times \dot{\boldsymbol{V}}_{0 \_2, i} \underline{\times} \boldsymbol{P}_{F i}+\boldsymbol{V}_{0 \_2, i} \underline{\times} \boldsymbol{V}_{0 \_2, i} \times \boldsymbol{P}_{F i} \quad$ according to Eq. (52).

The lengths, velocities, accelerations, and jerks of the actuators under the motion laws of the output platform in
Table 3 are simulated and drawn in Figure 14, Figure 15, Figure 16 and Figure 17, respectively. Based on the derivation relationships between them, the algorithms above are verified.

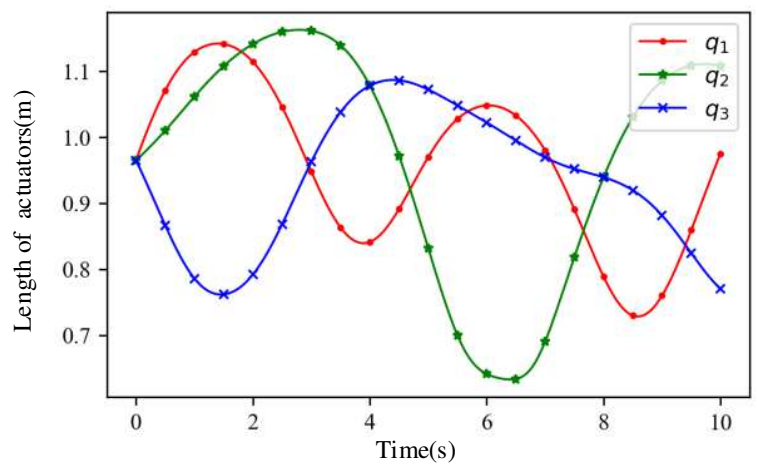

Figure 14 Lengths of actuators

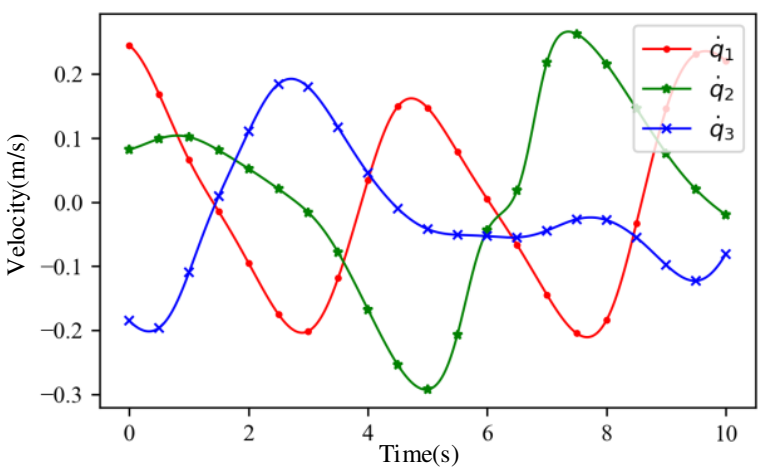

Figure 15 Velocities of actuators

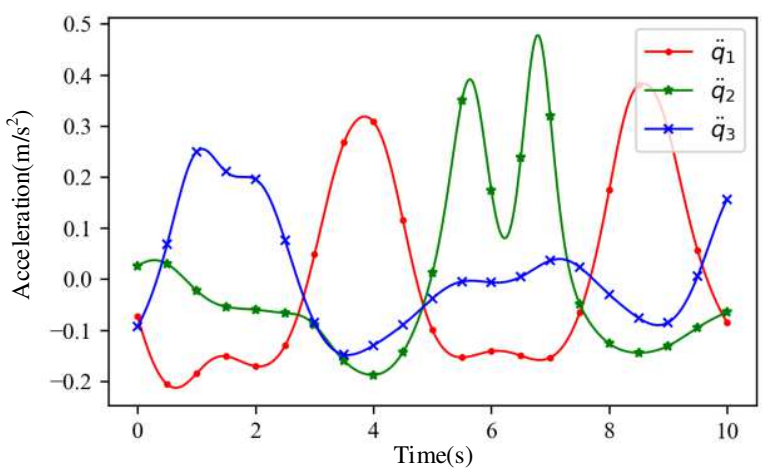

Figure 16 Accelerations of actuators 


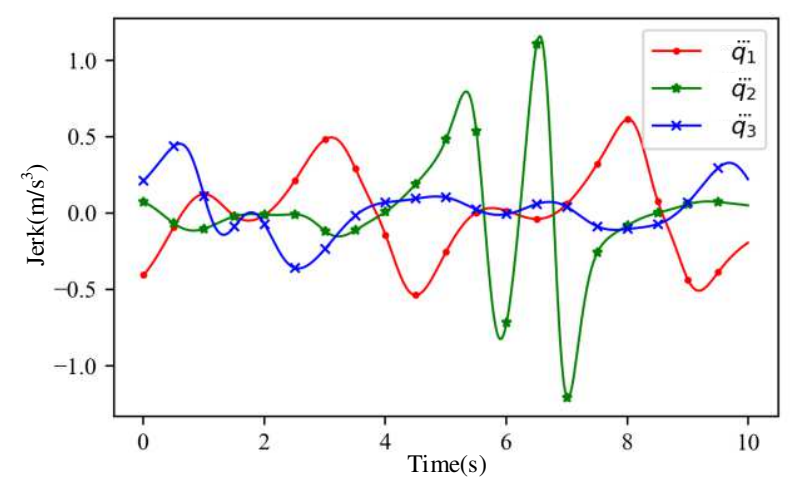

Figure 17 Jerks of actuators

\section{Conclusions}

This paper mainly solves higher-order kinematics modeling of serial and parallel mechanisms based on CGA representations and calculations. In order to frame rigid body and transform geometric entities between different frames, the mathematical relationships between flag and motor are given. The higher-order kinematics of serial chain mechanisms based on the motors and screws in CGA is built. The calculation methods of the position, velocity, acceleration, and jerk of the 3-RRS parallel mechanism are given and their correctness is verified by the calculating examples. It shows that CGA is more concise in expression, formula derivation, and calculation when applied in the higher-order kinematics of the 3-RRS parallel mechanism. The methods have potential applications in real-time motion planning, control, and computer simulation of mechanisms.

\section{Declaration}

\section{Acknowledgements}

\section{Funding}

Supported by National Natural Science Foundation of China (Grant No. 51875496), Pre-Research Project of the 13th-Five-Year-Plan on Common Technology (No. 41412040302), Science and Technology Planning Project of Hebei Province, China (Grant No. 18961828D), and Natural Science Foundation of Hebei Province of China(E2018404044).

\section{Availability of data and materials}

\section{Authors' contributions}

The author' contributions are as follows: Chang Wang wrote the manuscript; Tie-Shi Zhao was in charge of the whole trial; Er-Wei Li assisted with the programming. Yan-Zhi Zhao, Hui Bian and Ming-Chao Geng assisted with the basis theory of CGA.

\section{Competing interests}

The authors declare no competing financial interests.

\section{Consent for publication \\ Not applicable}

\section{Ethics approval and consent to participate}

Not applicable

\section{References}

[1] Thomas $\mathrm{M}$ and Tesar D. Dynamic Modeling of Serial Manipulator Arms. Journal of Dynamic Systems Measurement \& Control 1982; 104: 218.

[2] Freeman RA and Tesar D. The Generalized Coordinate Selection for the Dynamics of Complex Planar Mechanical Systems. Journal of Mechanical Design 1982; 104: 206.

[3] Osornio-Rios RA, de Jesús Romero-Troncoso R, Herrera-Ruiz G, et al. FPGA implementation of higher degree polynomial acceleration profiles for peak jerk reduction in servomotors. Robotics and Computer-Integrated Manufacturing 2009; 25: 379-392.

[4] Shieh R and Lu Y-S. Jerk-constrained time-optimal control of a positioning servo. In: ICCAS 20102010 , pp.1473-1476. IEEE.

[5] Macfarlane $\mathrm{S}$ and Croft EA. Jerk-bounded manipulator trajectory planning: design for real-time applications. IEEE Transactions on Robotics and Automation 2003; 19: 42-52.

[6] Haschke R, Weitnauer E and Ritter H. On-line planning of time-optimal, jerk-limited trajectories. In: 2008 IEEE/RSJ International Conference on Intelligent Robots and Systems 2008, pp.3248-3253. IEEE.

[7] Knežević B, Blanuša B and Marčetić D. Model of elevator drive with jerk control. In: 2011 XXIII International Symposium on Information, Communication and Automation Technologies 2011, pp.1-5. IEEE.

[8] Pendrill A-M. Rollercoaster loop shapes. Physics education 2005; 40: 517.

[9] Eager D, Pendrill A-M and Reistad N. Beyond 
velocity and acceleration: jerk, snap and higher derivatives. European Journal of Physics 2016; 37: 065008.

[10] Wohlhart K. Degrees of shakiness. Mechanism and Machine Theory 1999; 34: 1103-1126.

[11] Müller A. Recursive higher-order constraints for linkages with lower kinematic pairs. Mechanism and Machine Theory 2016; 100: 33-43. DOI: 10.1016/j.mechmachtheory.2016.01.012.

[12] Müller A. Higher derivatives of the kinematic mapping and some applications. Mechanism and Machine Theory 2014; 76: 70-85. DOI: 10.1016/j.mechmachtheory.2014.01.007.

[13] Müller A. Higher-Order Analysis of Kinematic Singularities of Lower Pair Linkages and Serial Manipulators. Journal of Mechanisms and Robotics 2018; 10. DOI: $10.1115 / 1.4038528$.

[14] Wu L, Müller A and Dai JS. A matrix method to determine infinitesimally mobile linkages with only first-order infinitesimal mobility. Mechanism and Machine Theory 2020; 148: 103776. DOI: 10.1016/j.mechmachtheory.2019.103776.

[15] Ball RS. A Treatise on the Theory of Screws. Cambridge, CA: Cambridge university press, 1900.

[16] Hunt KH. Kinematic geometry of mechanisms. USA: Clarendon Press Oxford, 1990.

[17] Waldron KJ. The constraint analysis of mechanisms. Journal of Mechanisms 1966; 1: 101-114.

[18] Sugimoto K and Duffy J. Applications of Linear Algebra to Screw Systems. Mechanism \& Machine Theory 1982; 17: 73-83.

[19] Duffy J. Statics and Kinematics with Applications to Robotics. Cambridge University Press, 1996.

[20] Phillips J. Freedom in machinery: Vol. 1 Introducing screw theory. Cambridge University Press, Cambridge, 1984.

[21] Selig JM. Geometric Fundamentals of Robotics. Springer Science \& Business Media, 2004.

[22] Dai J. Geometrical foundations and screw algebra for mechanisms and robotics. Beijing: Higher Education Press, 2014, ISBN:9787040334838 (translated from J. S. Dai, Screw Algebra and Kinematic Approaches for
Mechanisms and Robotics, Springer, London), p.30-90. [23] Huang Z, Li Q and Ding H. Theory of Parallel Mechanisms. Springer Netherlands, 2013.

[24] Mises R. Motorrechnung, ein neues Hilfsmittel der Mechanik. ZAMM- Journal of Applied Mathematics and Mechanics/Zeitschrift für Angewandte Mathematik und Mechanik 1924; 4: 155-181.

[25] Brand L. Vector and tensor analysis. New York: John wiley \& sons, 1947.

[26] Karger A and Novak J. Space kinematics and Lie groups. Hunter Publishing, Inc, 1985.

[27] Zhao T, Geng M, Chen Y, et al. Kinematics and dynamics Hessian matrices of manipulators based on screw theory. Chinese Journal of Mechanical Engineering 2015; 28: 226-235.

[28] Martinez JMR and Duffy J. An application of screw algebra to the acceleration analysis of serial chains. Mechanism and Machine Theory 1996; 31: 445-457. DOI: 10.1016/0094-114X(95)00089-H.

[29] Murray RM, Li Z and Sastry SS. A mathematical introduction to robotic manipulation. CRC press, 1994.

[30] Gallardo-Alvarado J. Kinematic Analysis of Parallel Manipulators by Algebraic Screw Theory. Cham: Springer International Publishing, 2016, p.99-132.

[31] Angles P. Construction de revêtements du groupe conforme d'un espace vectoriel muni d'une « métrique » de type (p, q). Annales De L Institut Henri Poincare-physique Theorique 1980; 33: 33-51.

[32] Angles P. Algebres De Clifford C r,s + Des Espaces Quadratiques Pseudo-Euclidiens Standards Er,s Et Structures Correspondantes Sur Les Espaces De Spineurs Associes. Plongements Naturels Des Quadratiques Projectives Reelles Q(Er,s) Attachees Aux Espaces Er,s. Springer Netherlands, 1986.

[33] Angles P. A Few Comments on Conformal Spin Structures and Conformal U(1)-Spin Structures on a Pseudo-Riemannian 2r-Dimensional Manifold V. Advances in Applied Clifford Algebras 2017; 27: 165-183. [34] Angles P. Obstruction Class for the Existence of a Conformal Spin Structure in a Strict Sense. Advances in Applied Clifford Algebras 2020; 30: 1-35.

[35] Hestenes D. New foundations for classical 
mechanics. Kluwer Academic, 2002.

[36] Li H, Hestenes D and Rockwood A. Generalized Homogeneous Coordinates for Computational Geometry. In: Sommer G (ed) Geometric Computing with Clifford Algebras: Theoretical Foundations and Applications in Computer Vision and Robotics. Berlin, Heidelberg: Springer Berlin Heidelberg, 2001, pp.27-59.

[37] Hestenes D. New Tools for Computational Geometry and Rejuvenation of Screw Theory. In: Bayro-Corrochano E and Scheuermann G (eds) Geometric Algebra Computing: in Engineering and Computer Science. London: Springer London, 2010, pp.3-33.

[38] Selig JM and Bayro-Corrochano E. Rigid Body Dynamics Using Clifford Algebra. Advances in Applied Clifford Algebras 2010; 20: 141-154. DOI: 10.1007/s00006-008-0144-1.

[39] Lasenby A, Lasenby R and Doran C. Rigid Body Dynamics and Conformal Geometric Algebra. Springer London, 2011.

[40] Berrondo M, Greenwald $\mathrm{J}$ and Verhaaren C. Unifying the inertia and Riemann curvature tensors through geometric algebra. American Journal of Physics 2012; 80: 905-912.

[41] Fu Z, Yang W and Yang Z. Solution of inverse kinematics for 6R robot manipulators with offset wrist based on geometric algebra. Journal of mechanisms and robotics 2013; 5: 0310081-0310087.

[42] Tichý R. Inverse Kinematics for the Industrial Robot IRB4400 Based on Conformal Geometric Algebra. In: International Conference on Modelling and Simulation for Autonomous Systesm 2019, pp.148-161. Springer.

[43] Aristidou A and Lasenby J. Inverse kinematics solutions using conformal geometric algebra. Guide to Geometric Algebra in Practice. Springer, 2011, pp.47-62.

[44] Huang X, Ma C and Su H. A geometric algebra algorithm for the closed-form forward displacement analysis of 3-PPS parallel mechanisms. Mechanism and Machine Theory 2019; 137: 280-296.

[45] Kim JS, Jeong JH and Park JH. Inverse kinematics and geometric singularity analysis of a 3-SPS/S redundant motion mechanism using conformal geometric algebra. Mechanism \& Machine Theory 2015; 90: 23-36.
[46] Li H and Zhang L. Line Geometry in Terms of the Null Geometric Algebra over $\mathbb{R}$ 3, 3, and Application to the Inverse Singularity Analysis of Generalized Stewart Platforms. Guide to Geometric Algebra in Practice. Springer, 2011, pp.253-272.

[47] Shen C, Hang L and Yang T. Position and orientation characteristics of robot mechanisms based on geometric algebra. Mechanism and Machine Theory 2017; 108: 231-243.

[48] Song Y, Han P and Wang P. Type synthesis of 1T2R and 2R1T parallel mechanisms employing conformal geometric algebra. Mechanism and Machine Theory 2018; 121: 475-486. DOI: 10.1016/j.mechmachtheory.2017.11.009.

[49] Yokomichi M, Takahashi N and Sato O. Modeling and Control Law Design for Rigid Body Dynamics by Conformal Geometric Algebra. In: society of instrument and control engineers of japan 2018, pp.653-660.

[50] Li Q, Chai X and Xiang J. Mobility Analysis of Limited-Degrees-of-Freedom Parallel Mechanisms in the Framework of Geometric Algebra. Journal of Mechanisms and Robotics 2016; 8: 041005.

[51] Chai X and Li Q. Analytical Mobility Analysis of Bennett Linkage Using Geometric Algebra. Advances in Applied Clifford Algebras 2017; 27: 2083-2095. DOI: 10.1007/s00006-017-0778-y.

[52] Serré P, Anwer N and Yang J. On the use of conformal geometric algebra in geometric constraint solving. Guide to Geometric Algebra in Practice. Springer, 2011, pp.217-232.

[53] Bayro-Corrochano E and Zamora-esquivel J. Differential and inverse kinematics of robot devices using conformal geometric algebra. Robotica 2007; 25: 43-61. DOI: $10.1017 / \mathrm{s} 0263574706002980$.

[54] Leo Dorst DFaSM. Geometric Algebra for Computer Science (Revised Edition). An Object-Oriented Approach to Geometry. Morgan Kaufmann: Elsevier Science, 2009.

[55] Perwass C, Edelsbrunner H, Kobbelt L, et al. Geometric algebra with applications in engineering. Berlin Heidelberg: Springer, 2009.

[56] Lavor C, Xambó-Descamps S and Zaplana I. A 
geometric algebra invitation to space-time physics, robotics and molecular geometry. Springer, 2018.

[57] hugohadfield, Wieser E, arsenovic a, et al. pygae/clifford v1.2.0. 2019.

[58] Hunt KH. Manipulating a body through a finite screw displacement. 1987.

[59] Hunt KH and Parkin IA. Finite displacements of points, planes, and lines via screw theory. Mechanism and Machine Theory 1995; 30: 177-192.

[60] Selig JM. Geometrical Methods in Robotics. Heidelberg: Springer, 1996.

\section{Biographical notes}

Chang Wang, born in 1988, is currently a $\mathrm{PhD}$ candidate at Parallel Robot and Mechatronic System Laboratory, Yanshan University, China. He received his master degree from Yanshan University, China, in 2014. His research interests include theory and application of parallel manipulators, and ship motion compensation technology.

E-mail: wangchangys@ stumail.ysu.edu.cn

Tie-Shi Zhao, born in 1963, is currently a professor and a PhD candidate supervisor at Parallel Robot and Mechatronic System Laboratory, Yanshan University, China. He received his $\mathrm{PhD}$ degree from Yanshan Universtiy, China, in 2000. His research interests include theory and application of parallel manipulators, wearable rehabilitation robot and ship motion compensation technology.

E-mail: tszhao@ysu.edu.cn

\section{Appendix}

The expressions of $A_{x}, A_{y}, A_{\alpha}$, and $B_{x}$ are listed as follows:
Er-Wei Li, born in 1987, is currently a lecturer at Parallel Robot and Mechatronic System Laboratory, Yanshan University, China. He received his $\mathrm{PhD}$ degree from Yanshan Universtiy, China, in 2018. His research interests include theory and application of parallel manipulators, and ship motion compensation technology. E-mail: lierwei@ysu.edu.cn

Yan-Zhi Zhao, born in 1981, is currently a professor and a PhD candidate supervisor at Parallel Robot and Mechatronic System Laboratory, Yanshan University, China. He received his $\mathrm{PhD}$ degree from Yanshan Universtiy, China, in 2009. His research interests include theory and application of parallel manipulators, and rescue searching and rescuing robot.

E-mail: yzzhao@ysu.edu.cn

Hui Bian, born in 1982, is currently an associate professor at Parallel Robot and Mechatronic System Laboratory, Yanshan University, China. He received his $\mathrm{PhD}$ degree from Yanshan Universtiy, China, in 2011. His research interests include theory and application of parallel manipulators, and wearable rehabilitation robot.

E-mail: ysubh@ysu.edu.cn

Ming-Chao Geng, born in 1984, is currently a lecturer at School of Mechanical Engineering, Hebei University of Architecture, China. He received his $\mathrm{PhD}$ degree from Yanshan Universtiy, China, in 2015. His research interests include parallel robots and multi-bodies system dyanmics.

E-mail: gmingchao@163.com

$$
\begin{aligned}
& B_{x}=\mathrm{s} \alpha+\gamma+\beta-\mathrm{s} \alpha-\gamma+\beta-\mathrm{s} \alpha+\gamma-\beta+\mathrm{s} \alpha-\gamma-\beta-2 \mathrm{c} \alpha-\gamma-2 \mathrm{c} \alpha+\gamma-2 \mathrm{c} \alpha-\beta-2 \mathrm{c} \alpha+\beta \\
& A_{x}=\mathrm{s} \alpha+\gamma+\beta \dot{\gamma} \mathrm{c} \alpha-\beta+\mathrm{s} \alpha+\gamma+\beta \dot{\gamma} \mathrm{c} \alpha+\beta+2 \mathrm{~s} \alpha+\gamma+\beta \dot{\beta} \mathrm{c} \alpha-\beta+ \\
& \text { s } \alpha-\gamma+\beta \dot{\gamma} \mathrm{c} \alpha-\beta+\mathrm{s} \alpha-\gamma+\beta \dot{\gamma} \mathrm{c} \alpha+\beta-2 \mathrm{~s} \alpha-\gamma+\beta \dot{\beta} \mathrm{c} \alpha-\beta- \\
& \text { s } \alpha+\gamma-\beta \dot{\gamma} \mathrm{c} \alpha-\beta-\mathrm{s} \alpha+\gamma-\beta \dot{\gamma} \mathrm{c} \alpha+\beta+2 \mathrm{~s} \alpha+\gamma-\beta \dot{\beta} \mathrm{c} \alpha+\beta- \\
& \text { s } \alpha-\gamma-\beta \dot{\gamma} \mathrm{c} \alpha-\beta-\mathrm{s} \alpha-\gamma-\beta \dot{\gamma} \mathrm{c} \alpha+\beta-2 \mathrm{~s} \alpha-\gamma-\beta \dot{\beta} \mathrm{c} \alpha+\beta+\text {. } \\
& \text { 2c } \alpha-\gamma \dot{\gamma} \mathrm{c} \alpha-\beta+2 \mathrm{c} \alpha-\gamma \dot{\gamma} \mathrm{c} \alpha+\beta-2 \mathrm{c} \alpha-\gamma \dot{\beta} \mathrm{c} \alpha-\beta+ \\
& 2 \mathrm{c} \alpha-\gamma \bar{j} \mathrm{c} \alpha+\beta>2 \mathrm{c} \alpha+\gamma\rangle \mathrm{c} \alpha-\beta>2 \mathrm{c} \alpha+\gamma\rangle \mathrm{c} \alpha+\beta\rangle \\
& 2 \mathrm{c} \alpha+\gamma \overline{\mathrm{D}} \mathrm{c} \alpha-\beta \rightarrow 2 \mathrm{c} \alpha+\gamma \overline{\mathrm{D}} \mathrm{c} \alpha+\beta) \\
& A_{\alpha}=\mathrm{s} \alpha+\gamma+\beta \dot{\gamma}+\mathrm{s} \alpha+\gamma+\beta \dot{\beta}+\mathrm{s} \alpha-\gamma+\beta \dot{\gamma}-\mathrm{s} \alpha-\gamma+\beta \dot{\beta}-\mathrm{s} \alpha+\gamma-\beta \dot{\gamma}+ \\
& \text { s } \alpha+\gamma-\beta \dot{\beta}-\mathrm{s} \alpha-\gamma-\beta \dot{\gamma}-\mathrm{s} \alpha-\gamma-\beta \dot{\beta}+2 \mathrm{c} \alpha-\gamma \dot{\gamma}-2 \mathrm{c} \alpha+\gamma \dot{\gamma}+ \\
& 2 \dot{\beta} \mathrm{c} \alpha-\beta-2 \dot{\beta} \mathrm{c} \alpha+\beta
\end{aligned}
$$

The expressions of the velocity of output platform $\boldsymbol{V}_{0_{-} H}$ are listed as follows: 
- 20 •

Jia-Fan Chang et al.

$$
\begin{aligned}
& V_{0_{-} H}=\boldsymbol{e}_{\infty} \dot{x} \boldsymbol{e}_{1}+\dot{y} \boldsymbol{e}_{2}+\dot{z} \boldsymbol{e}_{3}+\boldsymbol{T}_{0, H}-\dot{\alpha} \boldsymbol{e}_{1,2} \quad \tilde{\boldsymbol{T}}_{0, H}+\boldsymbol{T}_{0, H} \boldsymbol{R}_{z} \alpha \quad \dot{\beta} \boldsymbol{e}_{1,3} \tilde{\boldsymbol{R}}_{z} \alpha \tilde{\boldsymbol{T}}_{0, H} \\
& +\boldsymbol{T}_{0, H} \boldsymbol{R}_{z} \alpha \begin{array}{lllllllll}
\boldsymbol{R}_{y} & \beta & -\dot{\gamma} \boldsymbol{e}_{2,3} & \tilde{\boldsymbol{R}}_{y} & \beta & \tilde{\boldsymbol{R}}_{z} & \alpha & \tilde{\boldsymbol{T}}_{0, H}
\end{array} \\
& =-\dot{\alpha}+\mathrm{s} \beta \dot{\gamma} \boldsymbol{e}_{1,2}+\left(\frac{1}{2} \dot{\gamma} \mathrm{s} \alpha-\beta+\frac{1}{2} \dot{\gamma} \mathrm{s} \alpha+\beta+\dot{\beta} \mathrm{c} \alpha\right) e_{1,3}+\left(-\frac{1}{2} \mathrm{c} \alpha-\beta \dot{\gamma}-\frac{1}{2} \mathrm{c} \alpha+\beta \dot{\gamma}+\dot{\beta} \mathrm{s} \alpha\right) e_{2,3}+
\end{aligned}
$$

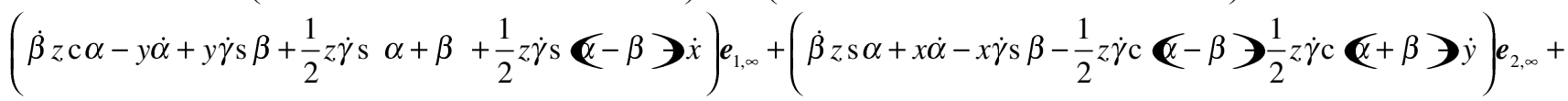

$$
\begin{aligned}
& \left(-\dot{\beta} y \mathrm{~s} \alpha+\frac{1}{2} y \dot{\gamma} \mathrm{c} \ll-\beta \rightarrow \frac{1}{2} y \dot{\gamma} \mathrm{c} \ll+\beta>\frac{1}{2} x \dot{\gamma} \mathrm{s} \ll+\beta>\frac{1}{2} x \dot{\gamma} \mathrm{s} \alpha-\beta \gg \dot{\beta} x \mathrm{c} \alpha-\dot{z}\right) e_{3, \infty}
\end{aligned}
$$

The angular velocities of joints from limb $i$ :

$$
\dddot{\boldsymbol{\theta}}_{0_{-} 5, i}=\mathbf{J}_{0_{-}, i, i}^{ \pm} \mathcal{S}\left({ }^{0} \dot{\overline{\boldsymbol{V}}}_{0_{-} H}-2 \sum_{i=1}^{n-1} \ddot{\theta}_{i_{-} i+1}{ }^{0} \boldsymbol{V}_{0_{-} i} \times{ }^{0} \overline{\boldsymbol{V}}_{i_{-} i+1}-\sum_{i=1}^{n-1} \dot{\theta}_{i_{-} i+1}{ }^{0} \dot{\boldsymbol{V}}_{0_{-} i} \times{ }^{0} \overline{\boldsymbol{V}}_{i_{-} i+1}+{ }^{0} \boldsymbol{V}_{0_{-} i} \times{ }^{0} \boldsymbol{V}_{0_{-} i} \times{ }^{0} \overline{\boldsymbol{V}}_{i_{-} i+1}\right) \text {. }
$$


Figures

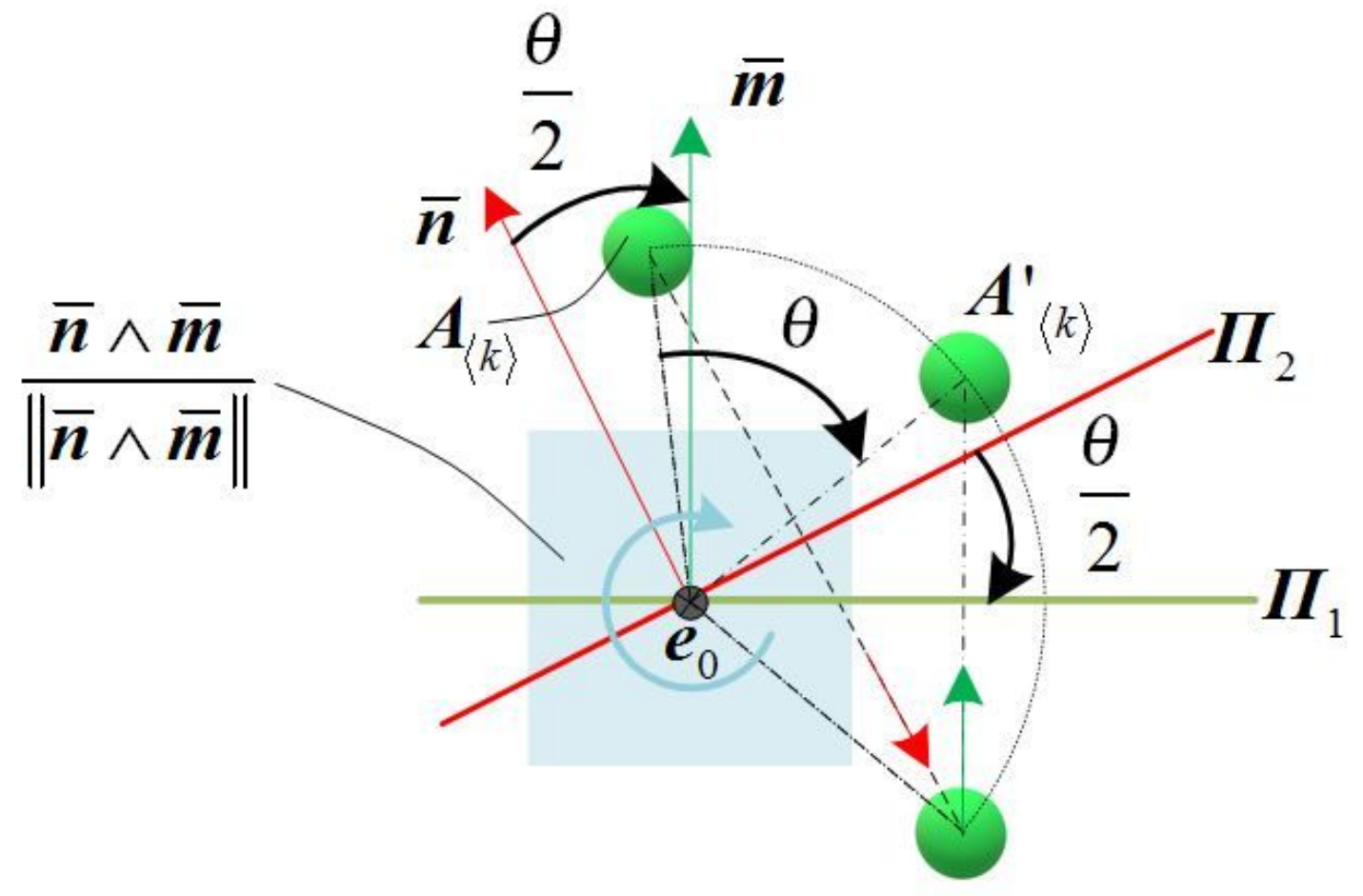

Figure 1

Rotation 


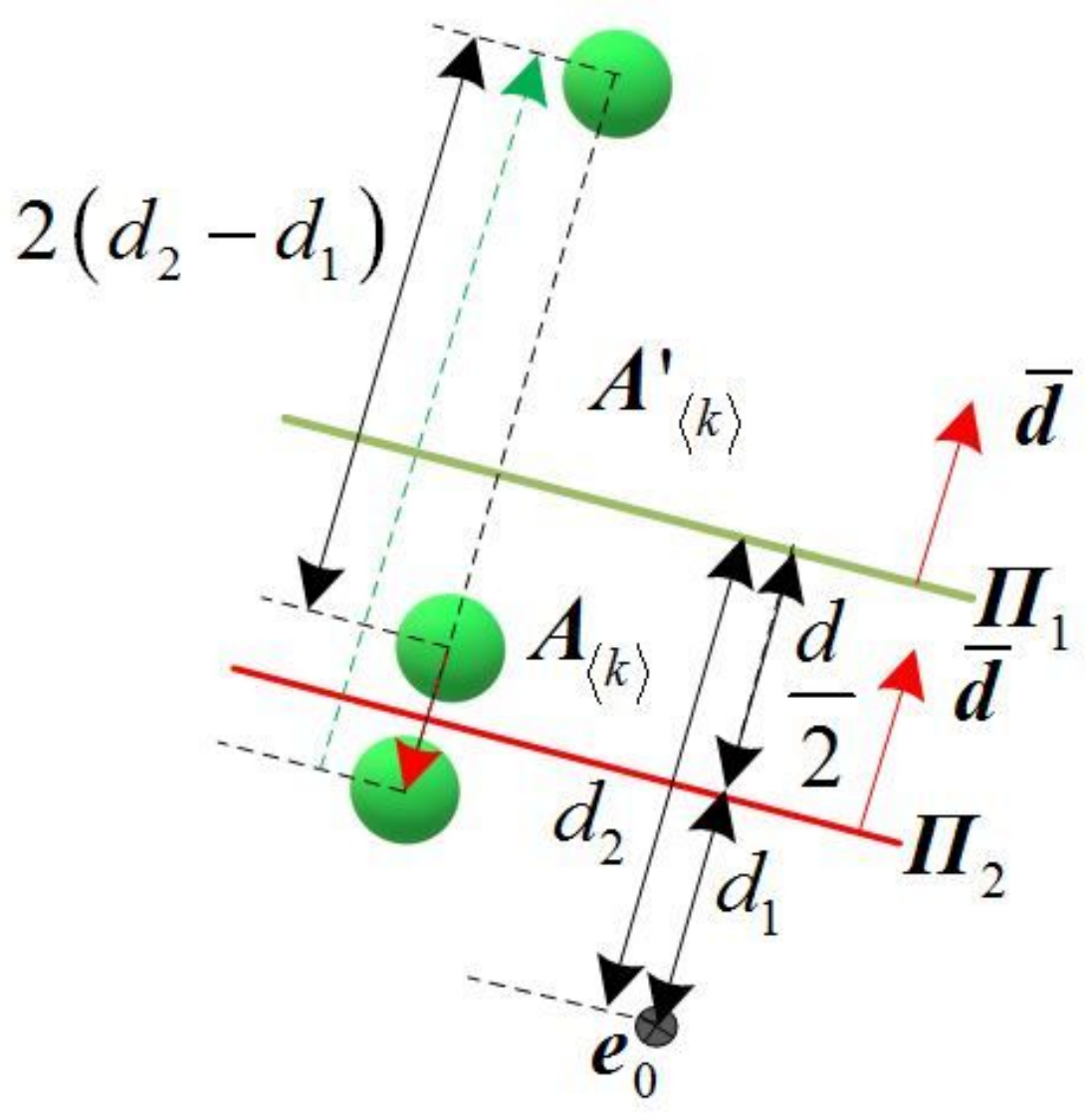

Figure 2

Translation 


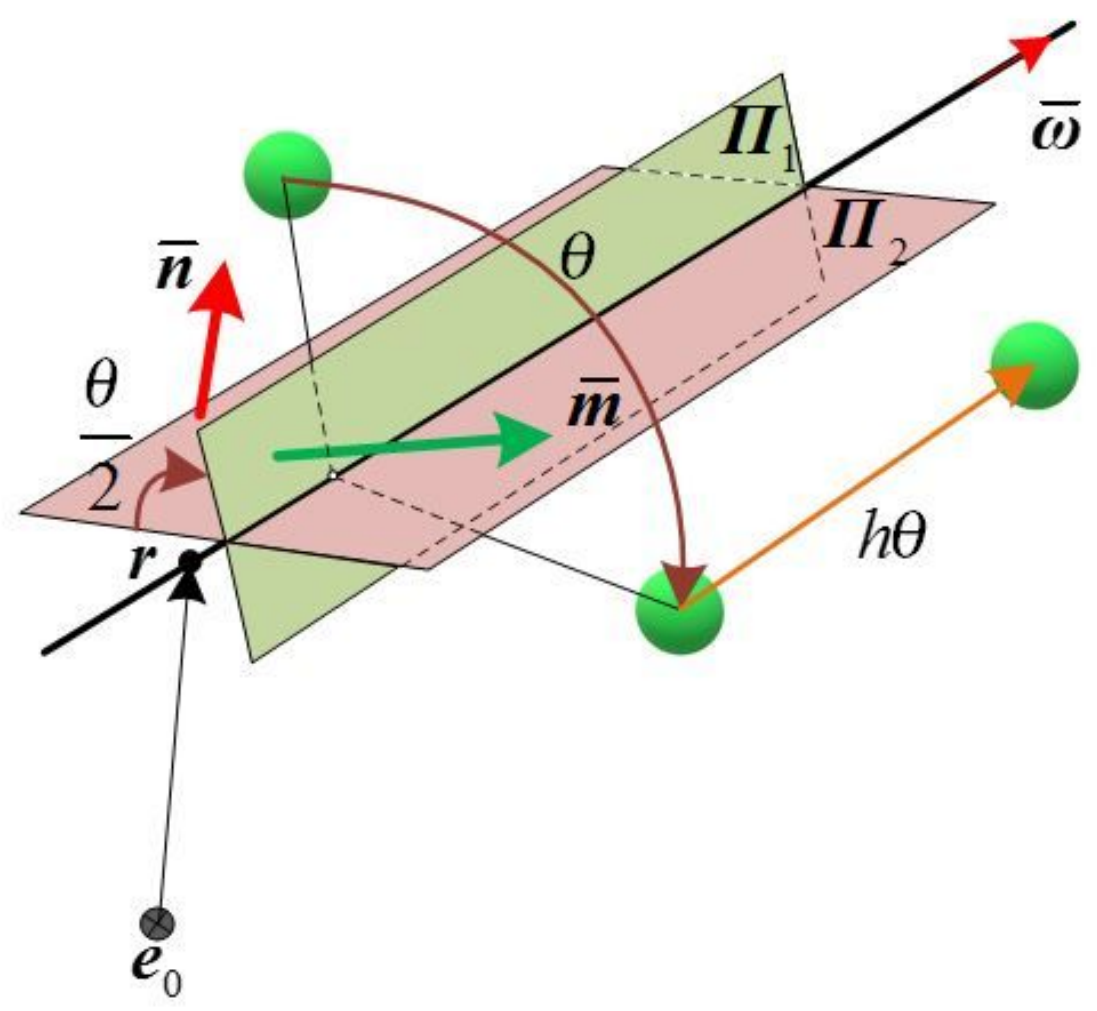

Figure 3

Screw motion

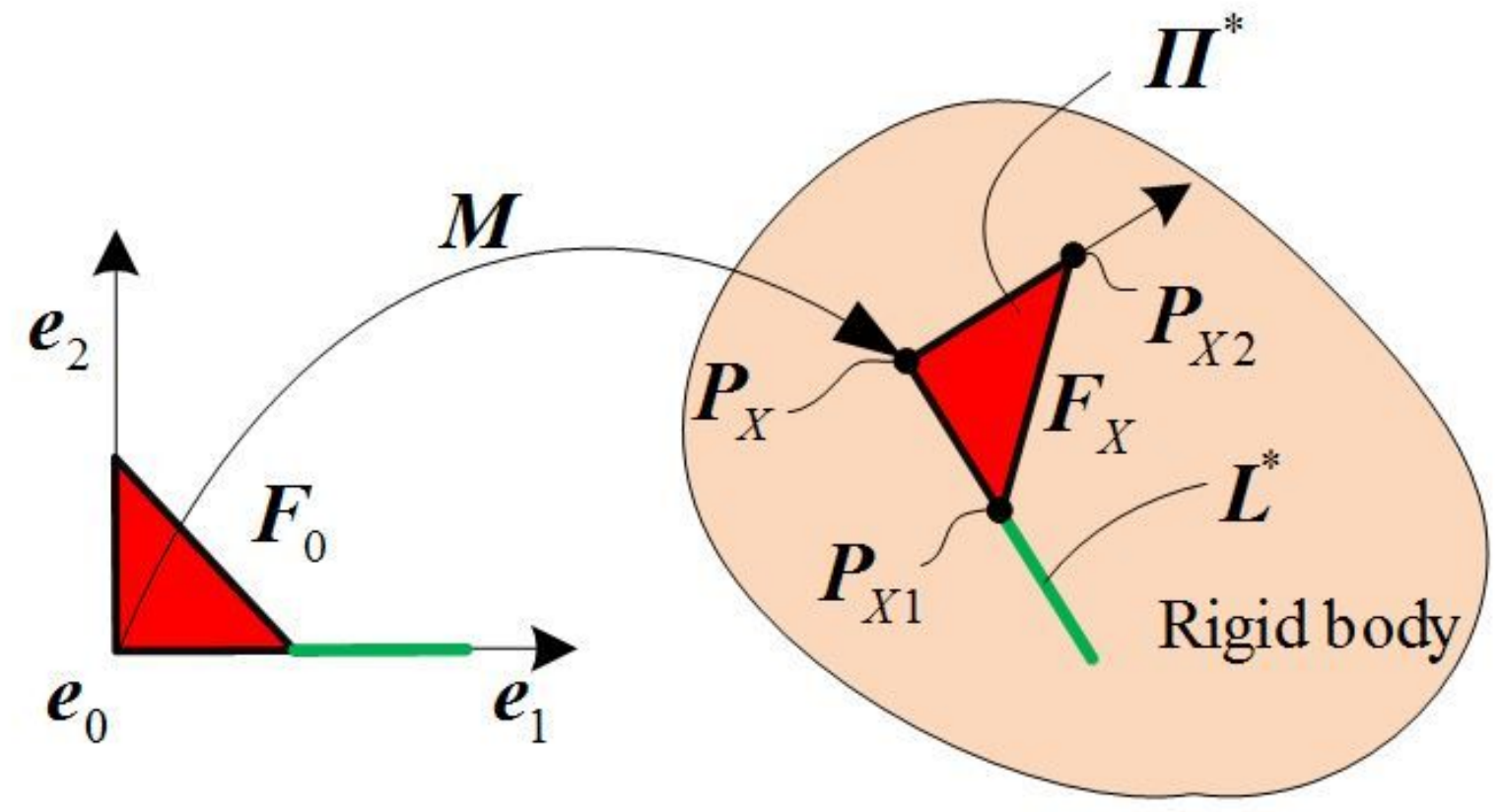

Figure 4 
Rigid body framing by flags

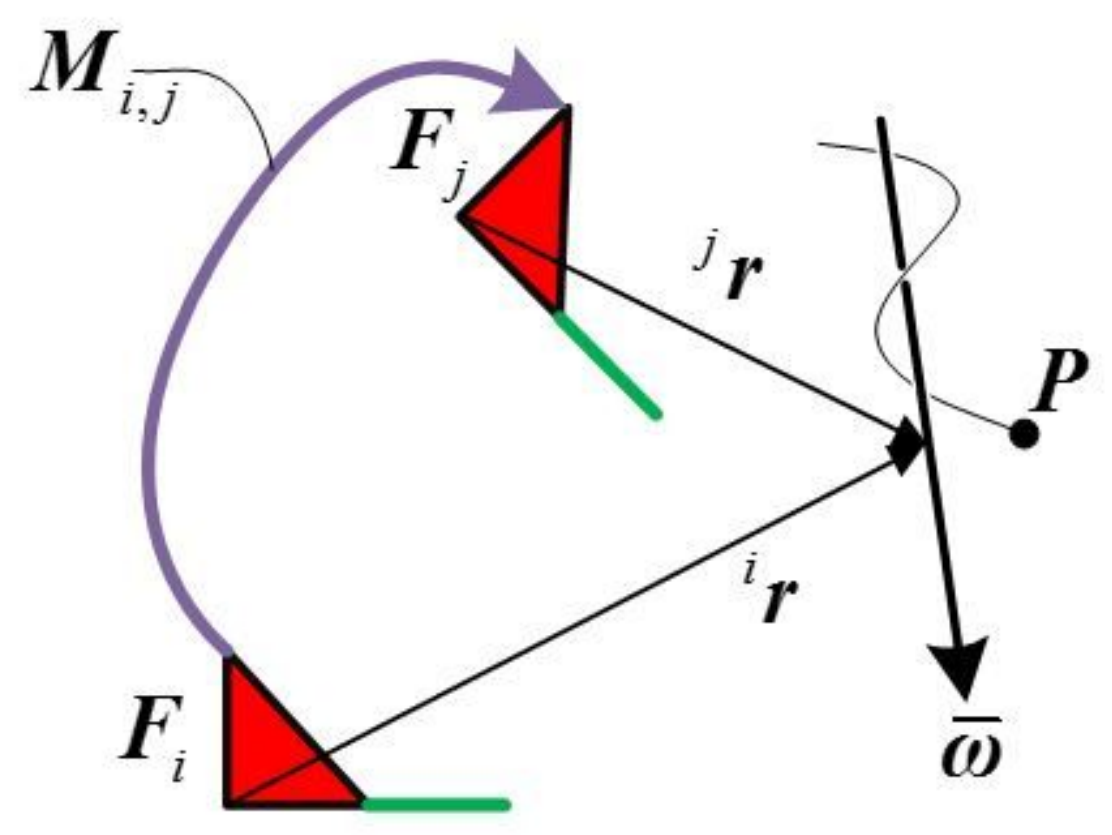

Figure 5

A screw in different frames

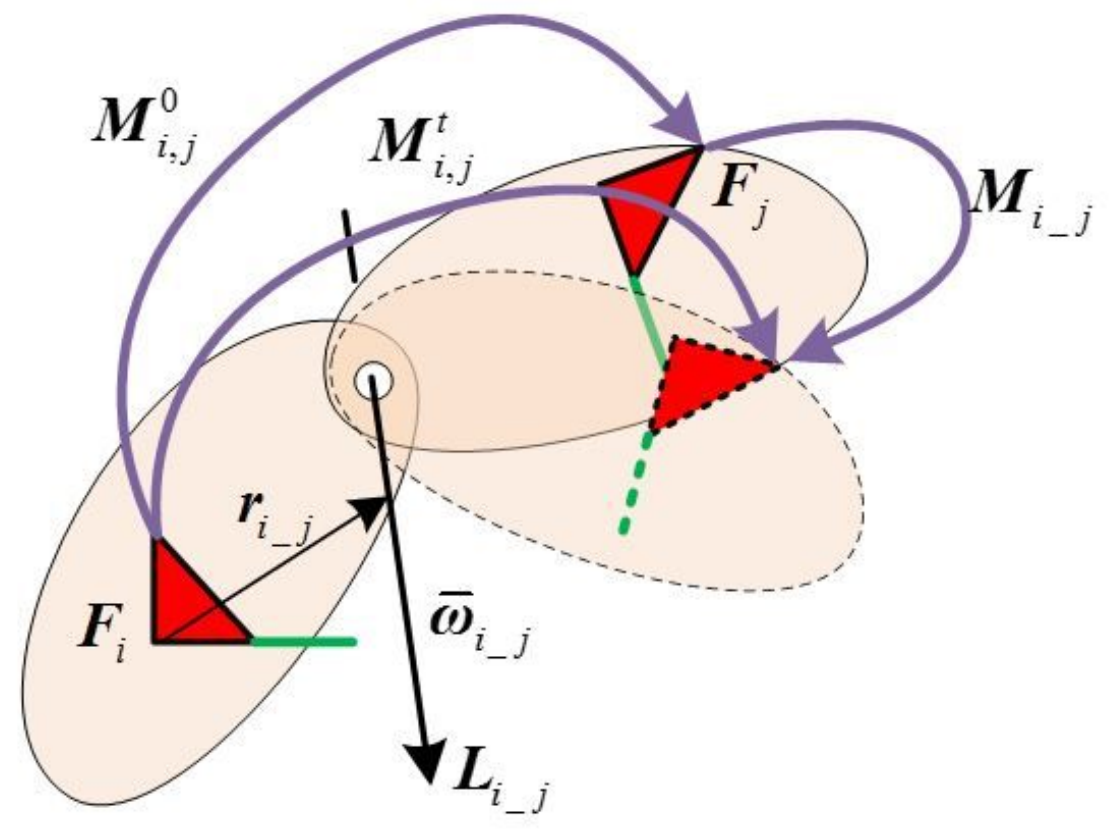

Figure 6

Single joint kinematics 


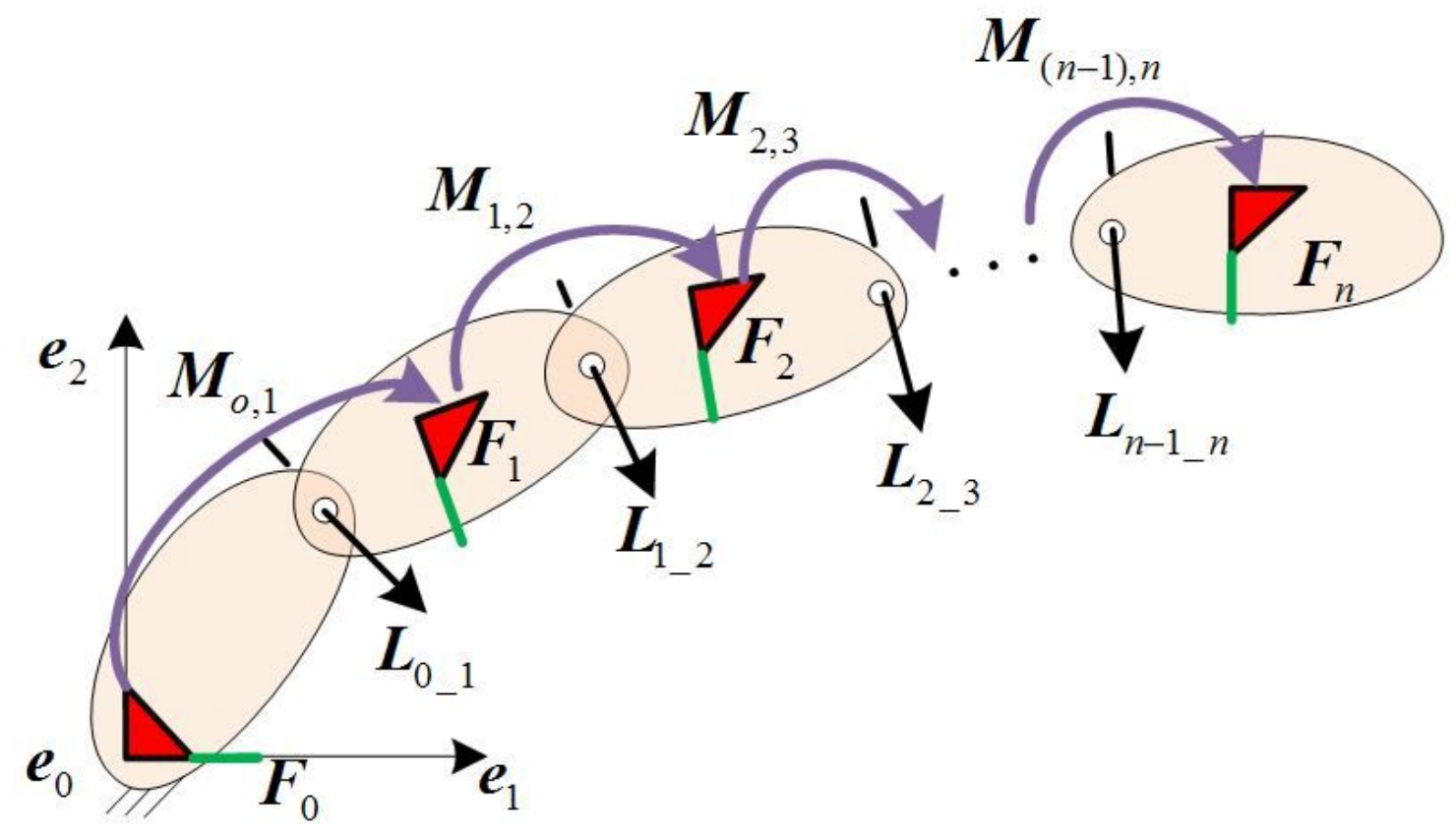

Figure 7

Kinematics of serial manipulator

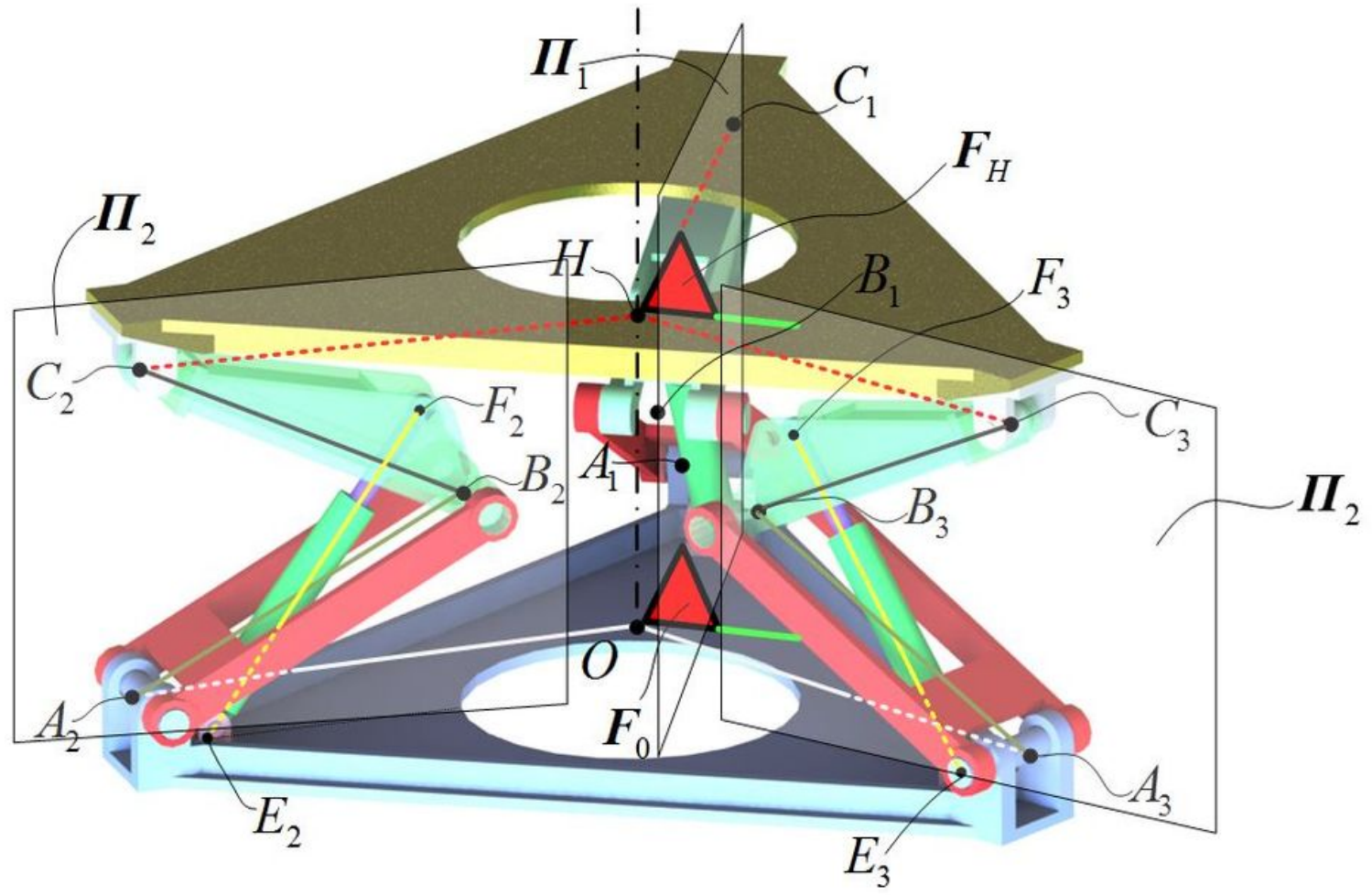

Figure 8

The 3-RRS mechanism 

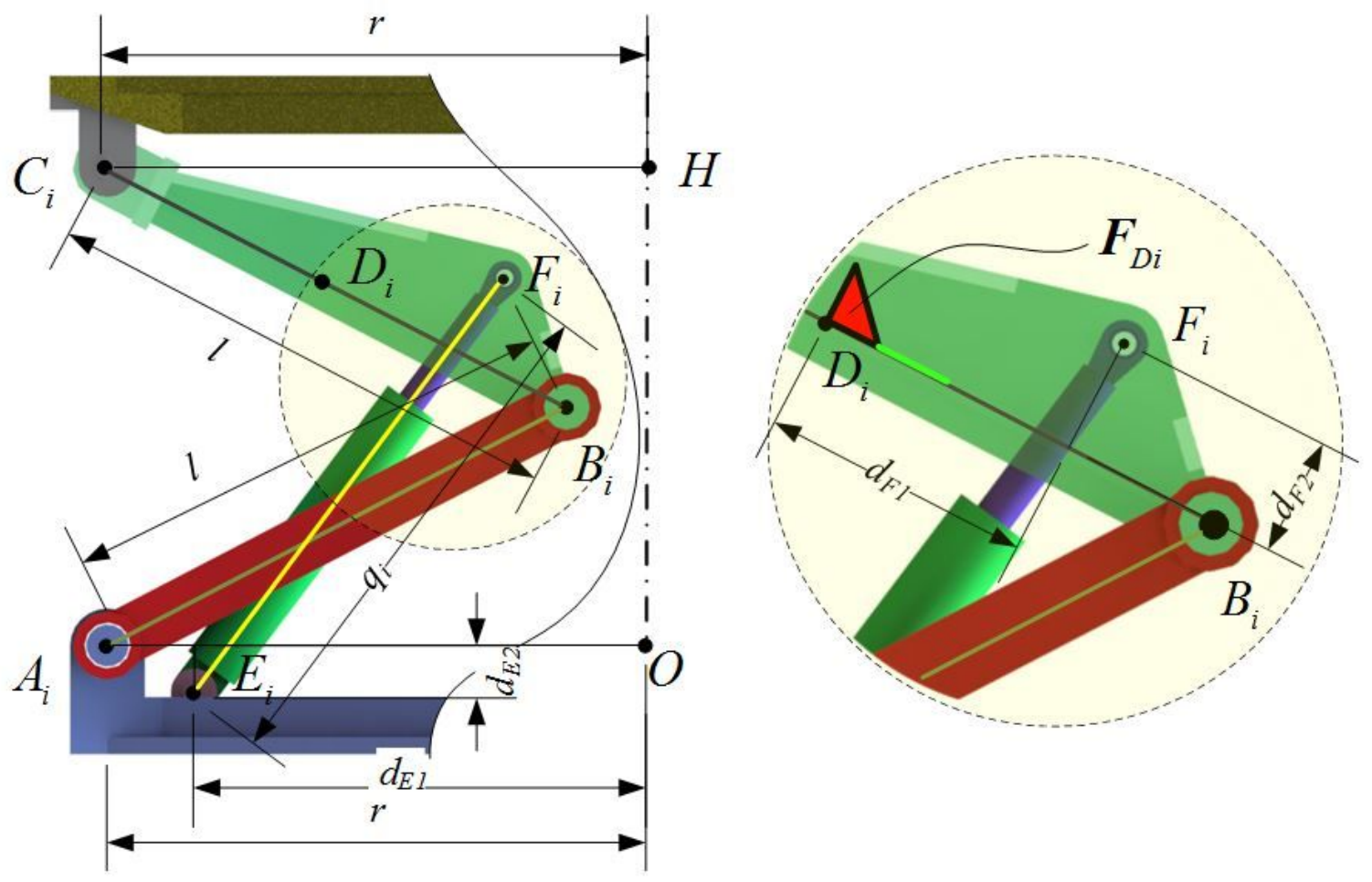

Figure 9

The geometry parameters of the i-th limb and actuator

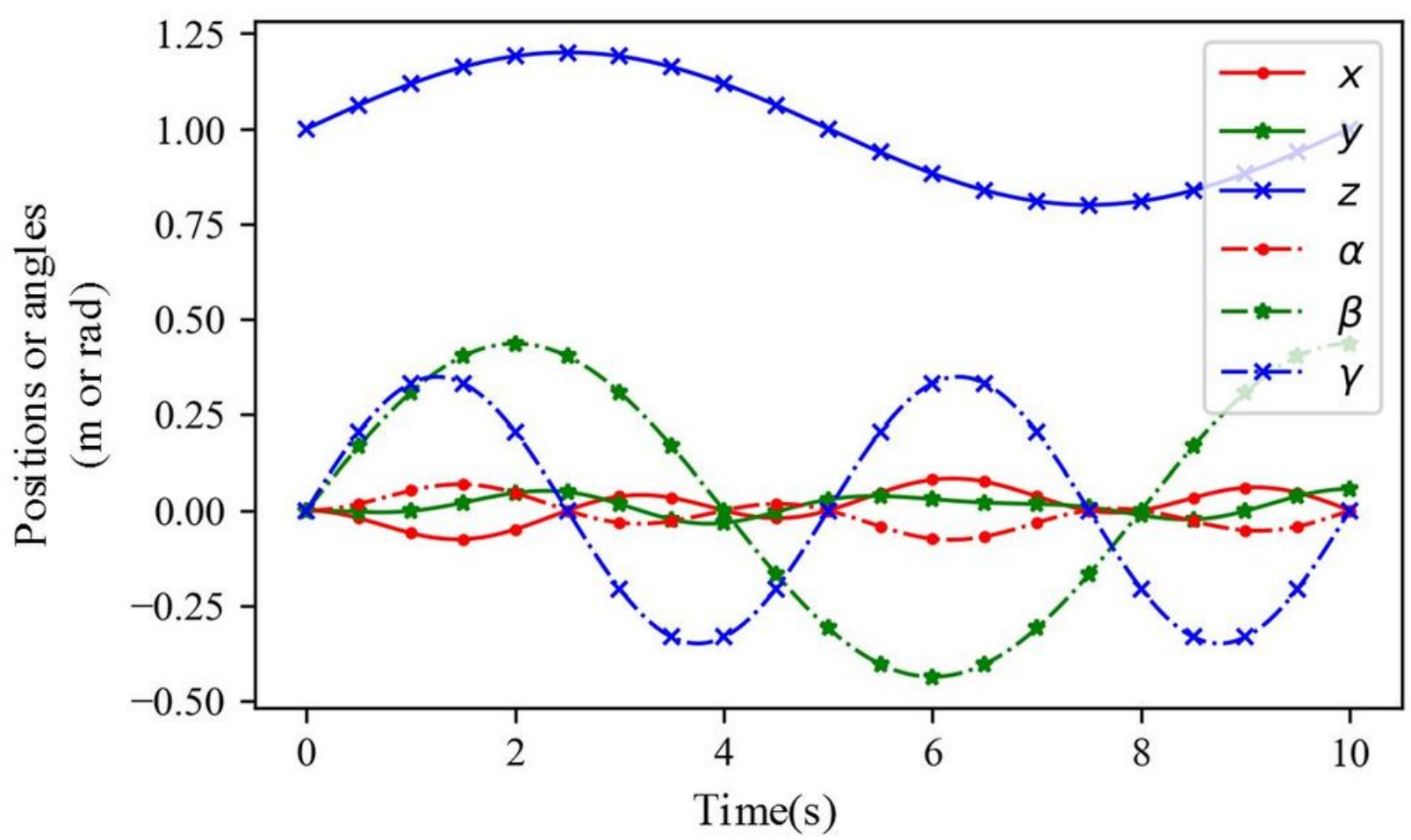

Figure 10 
Six DoF motions of the output platform

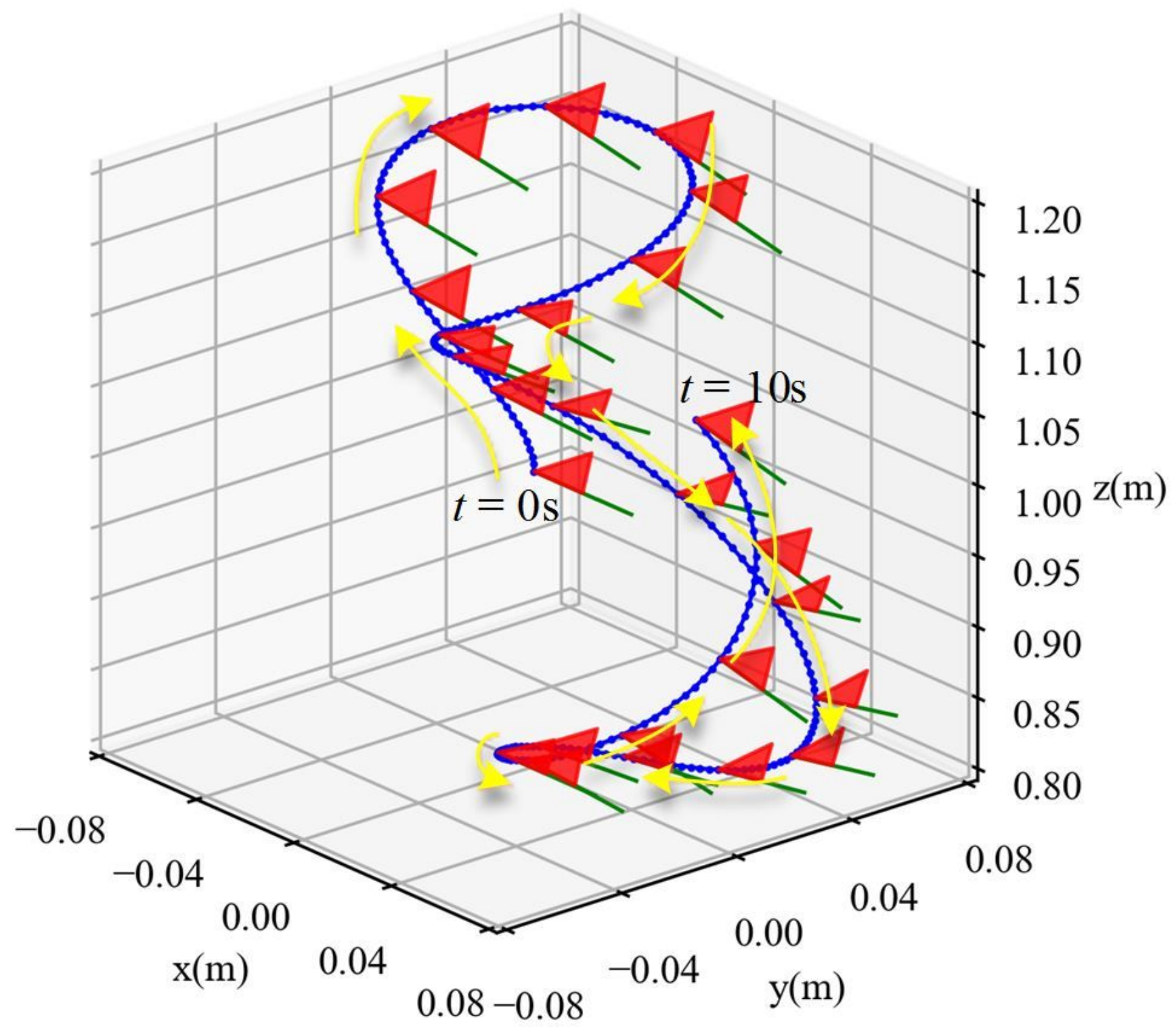

Figure 11

The trace of the flag attatched on the output platform from $0 \mathrm{~s}$ to $10 \mathrm{~s}$ 


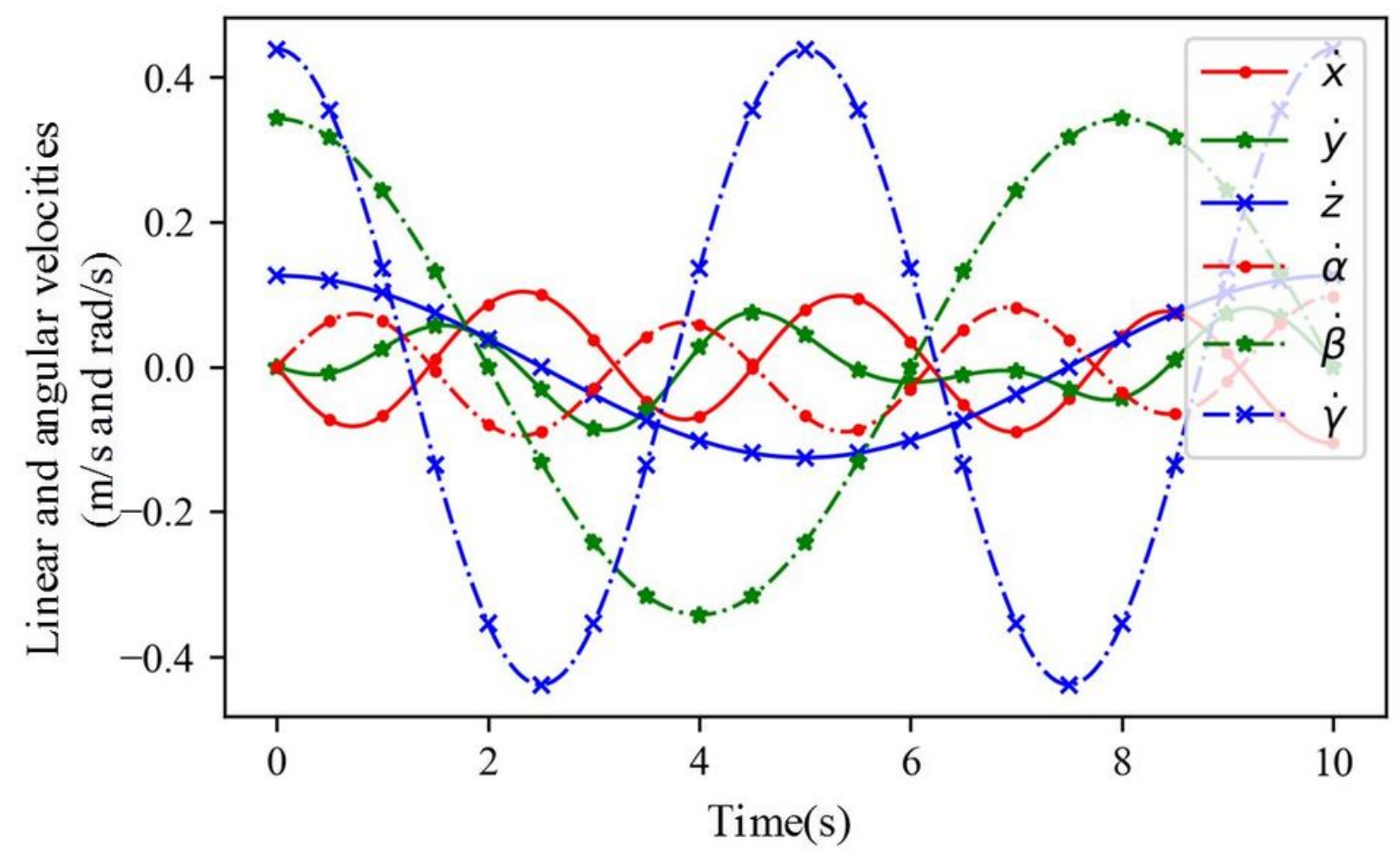

Figure 12

Velocity of the output platform 


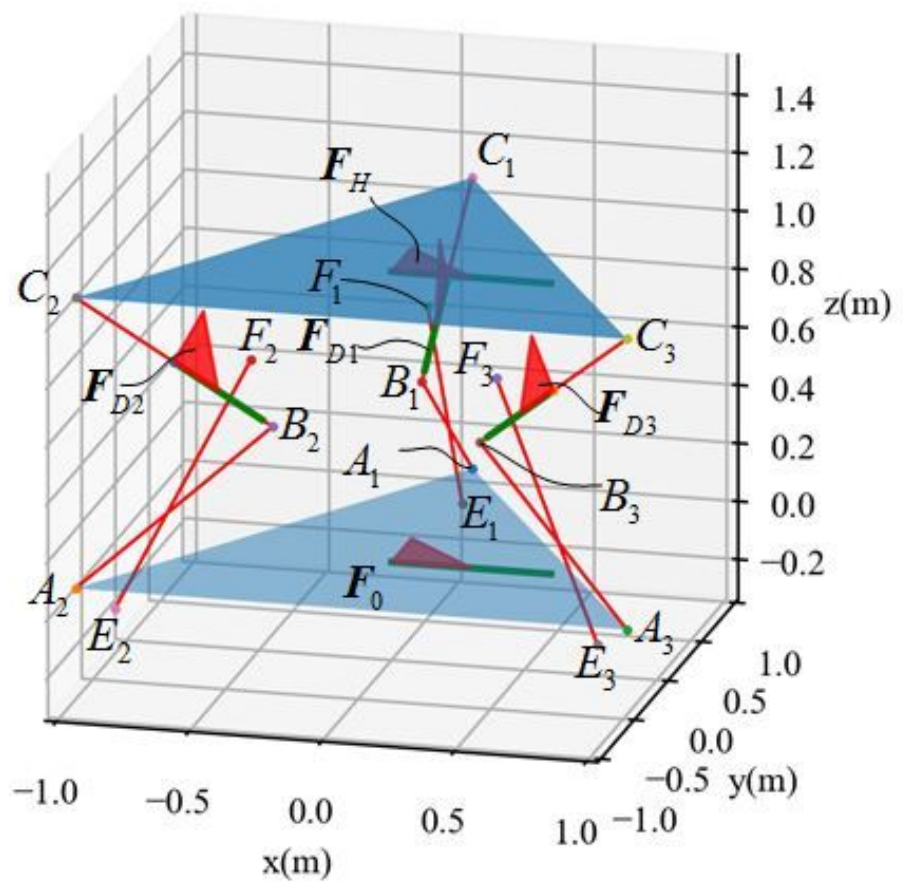

a) $z=1 \mathrm{~m}, \beta=0 \mathrm{deg}$, and $\gamma=0 \mathrm{deg}$

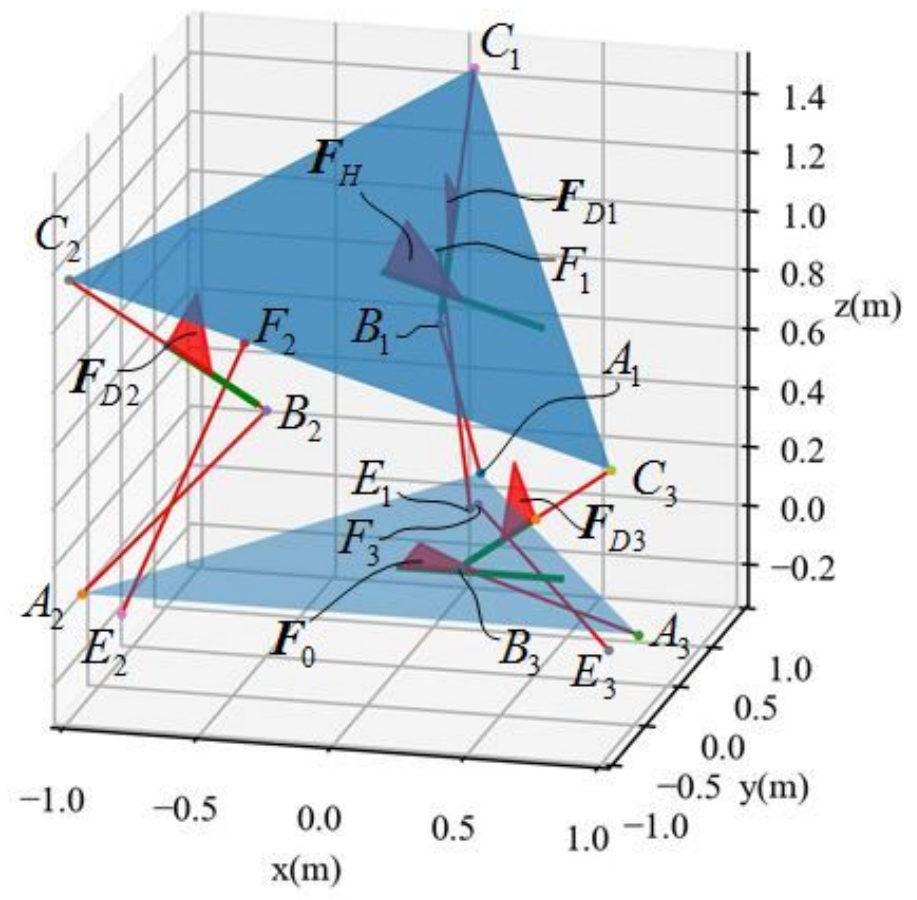

b) $z=1 \mathrm{~m}, \quad \beta=15 \mathrm{deg}$, and $\gamma=20 \mathrm{deg}$

\section{Figure 13}

Two configurations of the mechanism with points and flags 


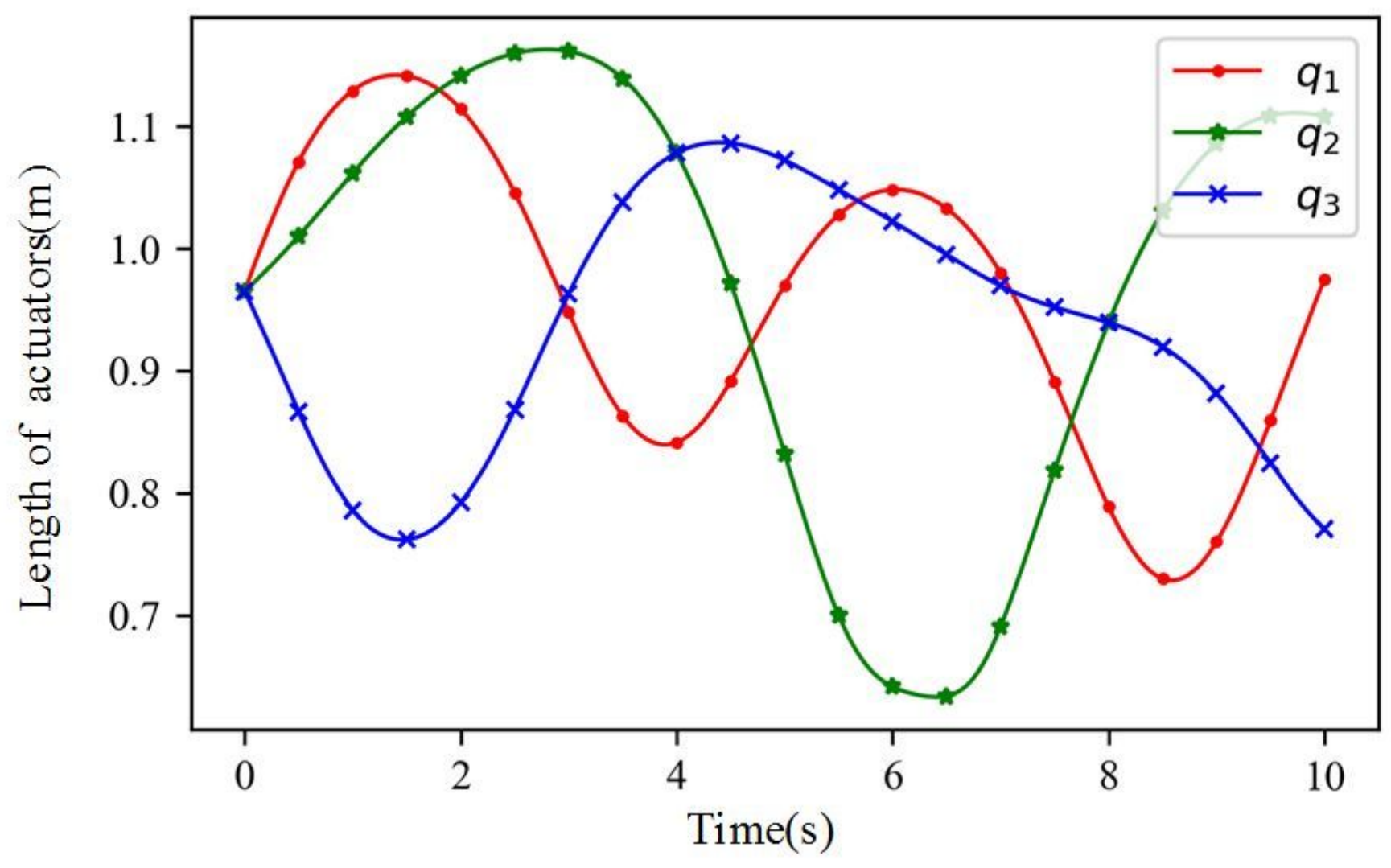

Figure 14

Lengths of actuators 


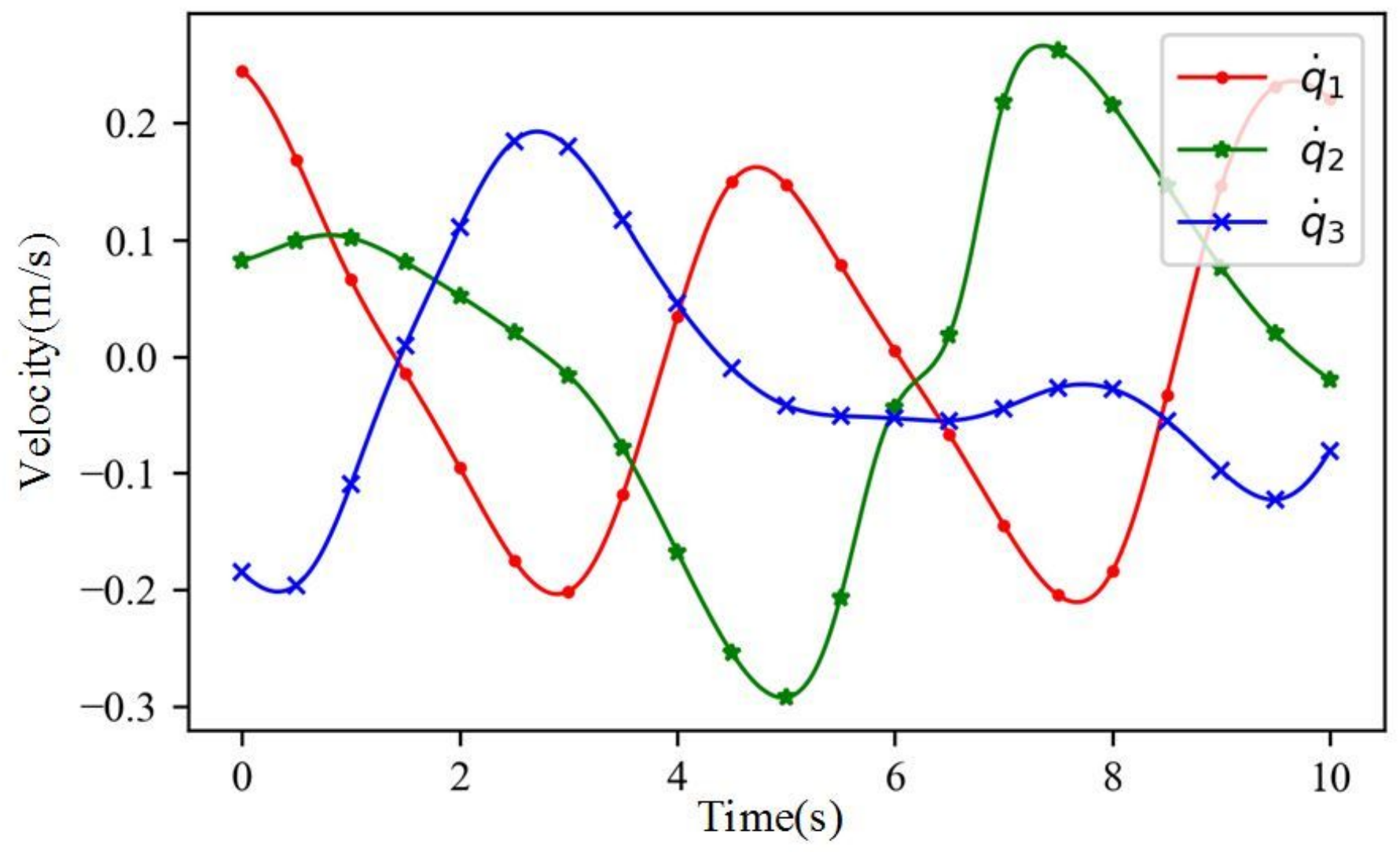

Figure 15

Velocities of actuators 


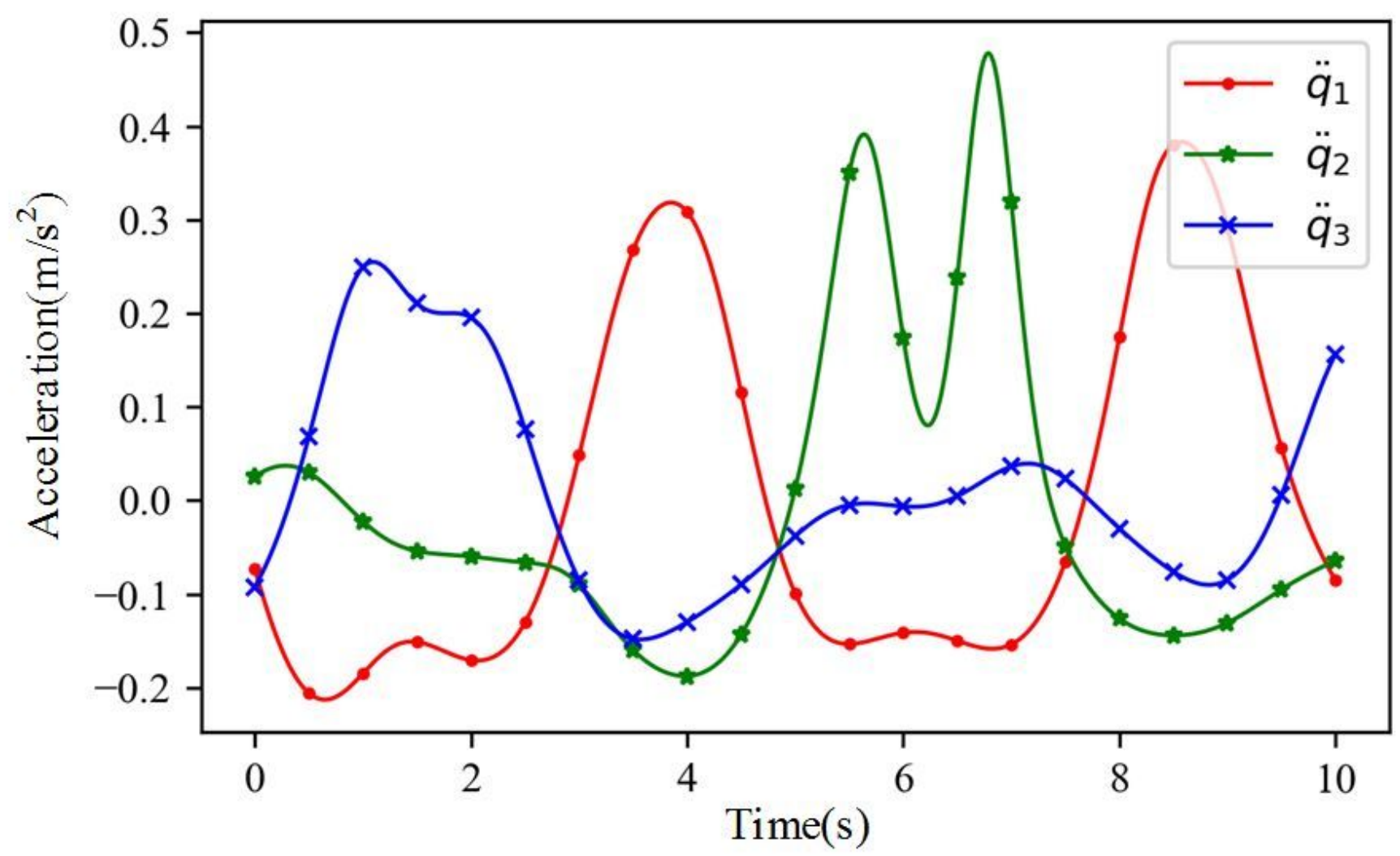

Figure 16

Accelerations of actuators 


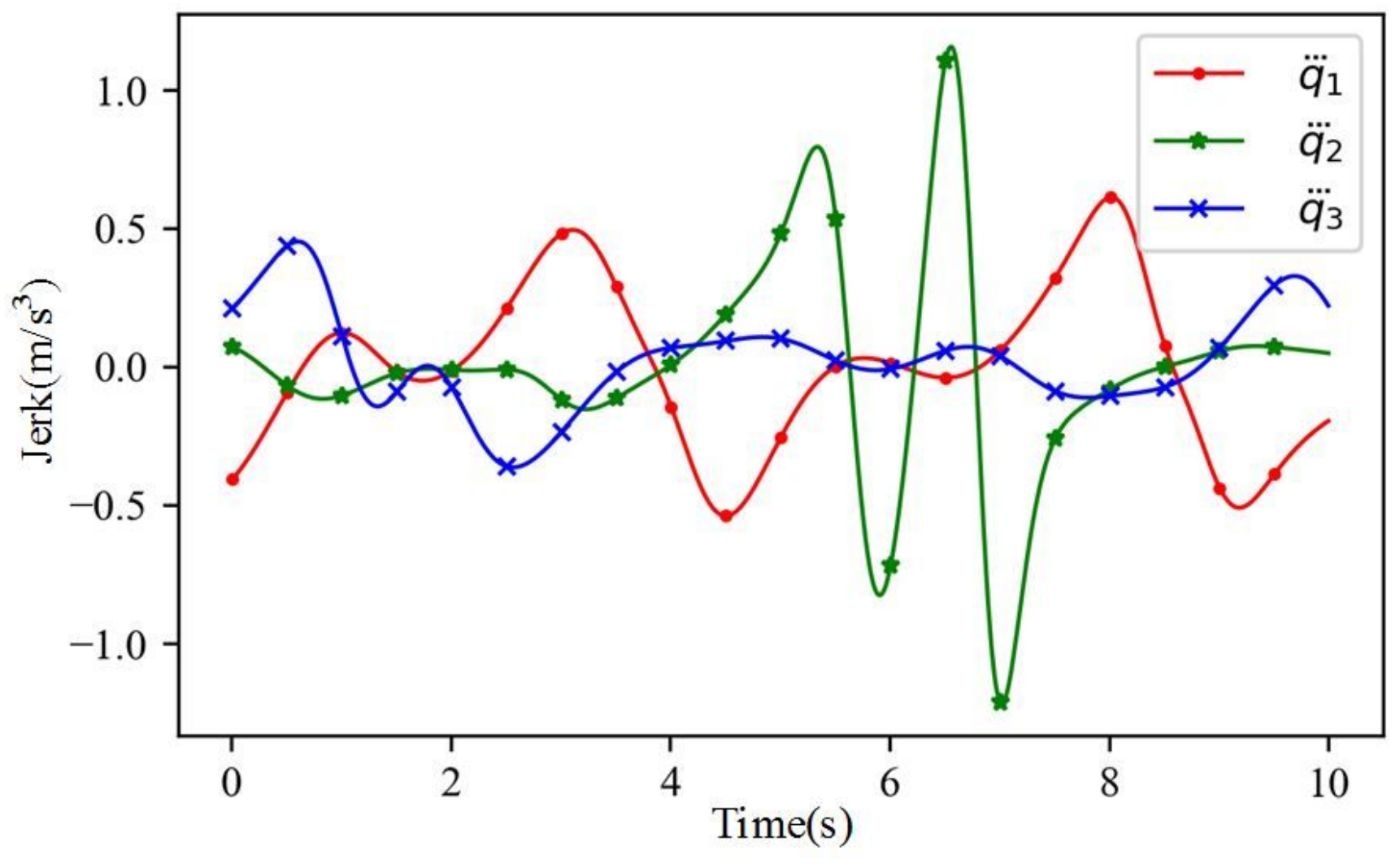

Figure 17

Jerks of actuators 